\title{
Risk-score model to predict prognosis of malignant airway obstruction after interventional bronchoscopy
}

\author{
Minlin Jiang ${ }^{1,2 \#}$, Hao Xu ${ }^{3 \#}$, Dongmei Yu ${ }^{4}$, Li Yang ${ }^{4}$, Wenhui $\mathrm{Wu}^{5}$, Hao Wang ${ }^{1,2}$, Hui Sun ${ }^{1}$, Jun Zhu ${ }^{1}$, \\ Wencheng Zhao ${ }^{1}$, Qiyu Fang ${ }^{1}$, Jia Yu ${ }^{1}$, Peixin Chen ${ }^{1,2}$, Shengyu Wu ${ }^{1,2}$, Zixuan Zheng ${ }^{1,2}$, Liping Zhang ${ }^{6}$, \\ Likun Hou ${ }^{6}$, Huixian Zhang ${ }^{7}$, Ye Gu${ }^{4}$, Yayi $\mathrm{He}^{1}$
}

${ }^{1}$ Department of Medical Oncology, Shanghai Pulmonary Hospital, Tongji University Medical School Cancer Institute, Tongji University School of Medicine, Shanghai, China; ${ }^{2}$ Tongji University, Shanghai, China; ${ }^{3}$ Department of Respiratory, the Second Affiliated Hospital of Zhejiang University, Hangzhou, China; ${ }^{4}$ Department of Endoscopy Center, Shanghai Pulmonary Hospital, Tongji University Medical School Cancer Institute, Tongji University School of Medicine, Shanghai, China; ${ }^{5}$ Pulmonary Hypertension Research Group, Quebec Heart and Lung Institute Research Centre (IUCPQ), Québec City, QC, Canada; ${ }^{6}$ Department of Pathology, Shanghai Pulmonary Hospital, Tongji University Medical School Cancer Institute, Tongji University School of Medicine, Shanghai, China; ${ }^{7}$ Department of Medical Oncology, The First Affiliated Hospital of Zhengzhou University, Zhengzhou, China

Contributions: (I) Conception and design: M Jiang, Y Gu, Y He; (II) Administrative support: Y Gu, Y He; (III) Provision of study materials or patients: H Xu, Y Gu, Y He; (IV) Collection and assembly of data: M Jiang, H Xu, D Yu, L Yang, L Zhang, H Wang, H Sun, J Zhu, W Zhao, L Hou; (V) Data analysis and interpretation: M Jiang, H Xu, W Wu, Q Fang, J Yu, P Chen, S Wu, Z Zheng, H Zhang; (VI) Manuscript writing: All authors; (VII) Final approval of manuscript: All authors.

\#These authors contributed equally to this work.

Correspondence to: Yayi He. Department of Medical Oncology, Shanghai Pulmonary Hospital, Tongji University Medical School Cancer Institute, Tongji University School of Medicine, No. 507 Zhengmin Road, Shanghai 200433, China. Email: 2250601@qq.com; Ye Gu. Department of Endoscopy Center, Shanghai Pulmonary Hospital, Tongji University Medical School Cancer Institute, Tongji University School of Medicine, No. 507 Zhengmin Road, Shanghai 200433, China. Email: Drsymons@outlook.com.

Background: Interventional bronchoscopy exhibits substantial effects for patients with malignant airway obstruction (MAO), while little information is available regarding the potential prognostic factors for these patients.

Methods: Between October 31, 2016, and July 31, 2019, a total of 150 patients undergoing interventional bronchoscopy and histologically-confirmed MAO were collected, in which 112 eligible participants formed the cohort for survival study. External validation cohort from another independent institution comprised 33 MAO patients with therapeutic bronchoscopy. The least absolute shrinkage and selection operator regression (LASSO) was applied to the model development dataset for selecting features correlated with MAO survival for inclusion in the Cox regression from which we elaborated the risk score system. A nomogram algorithm was also utilized.

Results: In our study, we observed a significant decline of stenosis rate after interventional bronchoscopy from $71.7 \% \pm 2.1 \%$ to $36.6 \% \pm 2.7 \%(\mathrm{P}<0.001)$ and interventional bronchoscopy dilated airway effectively. Patients in our study undergoing interventional bronchoscopy had a median survival time of 614.000 days (95\% CI: 269.876-958.124). Patients receiving distinct therapeutic methods of interventional bronchoscopy had different prognosis $(\mathrm{P}=0.022)$, and patients receiving treatment of electrocoagulation in combination with stenting and electrosurgical snare had worse survival than those receiving other options. Multivariate Cox analysis revealed that nonsmoking status, adenoid cystic carcinoma, and low preoperative stenosis length, as independent predictive factors for better overall survival (OS) of MAO patients. Then, the nomogram based on Cox regression and risk score system based on results from LASSO regression were elaborated respectively. Importantly, this risk score system was proved to have better performance than the nomogram and other single biomarkers such as traditional staging system (area under the curve 0.855 vs. $0.392-0.739)$. Survival curves showed that patients with the higher risk-score had poorer prognosis than 
those with lower risk-score (third quantile of OS: 126.000 days, 95\% CI: 73.588-178.412 vs. 532.000 days, 95\% CI: 0.000-1,110.372; $\mathrm{P}<0.001)$.

Conclusions: Nonsmoking status, adenoid cystic carcinoma, and low preoperative stenosis length, were independent predictive factors for better OS of MAO patients. We proposed a nomogram and risk score system for survival prediction of MAO patients undergoing interventional bronchoscopy with good performance.

Keywords: Lung cancer; malignant airway obstruction (MAO); therapeutic bronchoscopy; prognostic biomarker; risk score

Submitted Apr 14, 2021. Accepted for publication Jun 18, 2021.

doi: $10.21037 /$ tlcr-21-301

View this article at: https://dx.doi.org/10.21037/tlcr-21-301

\section{Introduction}

Malignant airway obstruction (MAO) is often a lifethreatening disorder with a 5 -year survival rate of $18.5 \%$, due to their debilitating dyspnea and subsequent diseases such as respiratory distress, infection, and bleeding (1). Stridor may occur in tracheobronchial stenosis less than $25 \%$ of the luminal diameter (2). The main cause of MAO is lung and esophageal cancers (3). In the course of patients' illness, thirty percent of patients with lung cancer were estimated to result in the obstruction of their trachea and principal bronchi (4).

Interventional bronchoscopy enables patency to maintain of airway, palliating symptoms immediately in these patients (5). Bronchoscopy options for these MAO patients include electrocautery (6), stenting (7), laser resection (8), argon plasma coagulation $(9,10)$, airway mechanical debridement and photodynamic therapy $(11,12)$. Measures such as high-frequency electric or argon plasma coagulation and laser photoresection should be considered in intraluminal tumors $(13,14)$. Stenosis caused by extrinsic compression of tumors or metastatic lymph nodes can be treated by airway stenting (15-17). However, although airway interventional treatments are effective in alleviating the clinical symptoms of patients with central airway stenosis $(2,18)$, accompanying complications are common. In patients with stent implantation, granulation tissue hyperplasia, stent displacement, stent fracture, phlegm thrombus formation and other complications such as atelectasis and infection occur frequently (19-21). In addition, secondary to malignancies, the underlying diseases of these patients are usually critical. Therefore, their prognosis is often not satisfactory in clinical practice. More supporting evidence of the efficacy of treatments on MAO patients is needed to better understand their prognosis. Moreover, exploring prognosis and potential prognostic risk factors among these populations can better procedure optimization and personalized clinical management. In this context, we first analyzed the short and intermediate efficacy of MAO patients undergoing interventional bronchoscopy. Then, the impacts of different interventional therapies and other clinical indicators on patients' prognosis were explored. By machine learning and statistical analysis, the risk-score and nomogram model were further constructed after repeated validations. Relevant data was also collected from another institute as an external dataset for verification. We present the following article in accordance with the TRIPOD reporting checklist (available at https://dx.doi. org/10.21037/tlcr-21-301).

\section{Methods}

\section{Data collection and cohort development}

We retrospectively identified a total of 240 patients who underwent interventional bronchoscopy at Shanghai Pulmonary Hospital between October 31, 2016, and July 31, 2019 and included 150 patients who had histologicallyconfirmed MAO (Table S1). Data screening excluded $52(34.7 \%)$ with missing data and indicated 98 (65.3\%) patients were available for analyzing the short and intermediate benefits of therapeutic bronchoscopy for MAO (Table S2). As for long-term prognosis evaluation, complete follow-up data of 112 patients were collected for survival analysis. After removing 8 patients with unspecific pathological types and 2 patients with histology of large cell neuroendocrine carcinoma and sarcomatoid carcinoma, 102 patients were available for model construction (Table 1, Figure 1). 
Two experienced pathologists (ZLP, HLK) further evaluated and verified these primary diagnoses. Histologically-confirmed MAO patients over 18 years old who received bronchoscopy were included. Adenoid cystic carcinoma is classified as a type of non-small cell lung cancer (NSCLC), but quite different from NSCLC in several aspects (22). Adenoid cystic carcinoma mainly grows in tracheal lumen, with a relatively slower growth rate. It rarely develops metastasis to lymph node and other distant organs even in the advanced stages (22). Therefore, a TNM staging method was proposed by Bhattacharyya (23). In our study, we used Bhattacharyya staging criteria for adenoid cystic carcinoma (23) and the eighth edition of TNM classification for squamous cell carcinoma (SCC), adenocarcinoma (ADC), and small cell carcinoma (small cell lung cancer, SCLC) of lung cancer (24). No esophagus cancer patient was enrolled in our study. The main exclusion criteria were cases $<18$ years old, pathologically-confirmed benign airway stenosis, having not received treatment of interventional bronchoscopy, and with inadequate baseline clinical characteristics. The major basic clinical features included gender, age, smoking history, clinical stage, pathological category, operative site, airway stenosis classification, specific method of the interventional bronchoscopy, treatments including chemotherapy, radiotherapy, immunotherapy, and targeted therapy after interventional bronchoscopy, receiving interventional bronchoscopy again due to restenosis, survival status, and overall survival (OS). OS was calculated from the specific date of interventional bronchoscopy to patients' death resulted from any reason. Short and intermediate benefits were defined radiologically as reopening patients' airway lumens to more than $50 \%$ of normal diameters. This study was conducted consistent with the provisions of Declaration of Helsinki (as revised in 2013). Approval from the ethics committee of Shanghai Pulmonary Hospital was obtained $(18 \mathrm{Q} 016 \mathrm{NJ})$ and individual consent for this retrospective analysis was waived.

\section{External data and validation cohort}

Between January 6, 2016 and May 17, 2019, a total of $33 \mathrm{MAO}$ patients undergoing bronchoscopy, with complete clinical information and the same clinical factors as our cohort for model development, were included as the validation cohort from the Second Affiliated Hospital of Zhejiang University, China. Patients in the validation cohorts were aged $>18$ years old and received therapies based on the protocol consistent with the development cohort. All of these patients had complete data for preoperative and postoperative computerized tomography (CT) scans. Short-period efficacy (<30 days) of bronchoscopy was verified. After follow-up, $24(24 / 33,72.7 \%)$ patients were available for survival analysis (Figure 1).

Table 1 Clinical characteristics of the cohort for survival study and model construction

\begin{tabular}{lcr}
\hline Clinical characteristics & Survival analysis dataset $(\mathrm{n}=112)$ & Model construction dataset $(\mathrm{n}=102)$ \\
\hline Age, median (range) & $62(26-89)$ & $62(26-89)$ \\
Sex, $\mathrm{n}(\%)$ & $92(82.1)$ & $85(83.3)$ \\
Male & $20(17.9)$ & $17(16.7)$ \\
Female & & $46(45.1)$ \\
Smoking history, $\mathrm{n}(\%)$ & $51(45.5)$ & $56(54.9)$ \\
Current/former & $61(54.5)$ & $2(2.0)$ \\
Never & & $70(68.6)$ \\
Clinical stage, $\mathrm{n}(\%)$ & $2(1.8)$ & $30(29.4)$ \\
Stage II & $76(67.9)$ & $34(30.4)$ \\
Stage III & & 30 \\
Stage IV & & \\
\hline
\end{tabular}

Table 1 (continued) 
Table 1 (continued)

\begin{tabular}{|c|c|c|}
\hline Clinical characteristics & Survival analysis dataset $(n=112)$ & Model construction dataset $(n=102)$ \\
\hline \multicolumn{3}{|l|}{ Histopathology, n (\%) } \\
\hline Squamous cell carcinoma & $66(58.9)$ & $65(63.7)$ \\
\hline Adenocarcinoma & $22(19.6)$ & $21(20.6)$ \\
\hline Small cell lung cancer & $6(5.4)$ & $6(5.9)$ \\
\hline Sarcomatoid carcinoma & $1(0.9)$ & $0(0.0)$ \\
\hline Unspecific pathological types & $8(7.1)$ & $0(0.0)$ \\
\hline \multicolumn{3}{|l|}{ Interventional surgery, n (\%) } \\
\hline Electrosurgical snare & $1(0.9)$ & $1(1.0)$ \\
\hline Combined therapy & $34(30.4)$ & $28(27.5)$ \\
\hline \multicolumn{3}{|l|}{ Lesion site*, n (\%) } \\
\hline Trachea & $35(31.3)$ & $32(31.4)$ \\
\hline Carina & $14(12.5)$ & $12(11.8)$ \\
\hline Right principal bronchus & $40(35.7)$ & $36(35.3)$ \\
\hline Right middle bronchus & $14(12.5)$ & $11(10.8)$ \\
\hline Left principal bronchus & $33(29.5)$ & $32(31.4)$ \\
\hline \multicolumn{3}{|c|}{ Respiratory failure before bronchoscopy, n (\%) } \\
\hline Yes & $2(1.8)$ & $2(2.0)$ \\
\hline No & $110(98.2)$ & $100(98.0)$ \\
\hline \multicolumn{3}{|c|}{ Treatment after interventional bronchoscopy, n (\%) } \\
\hline Chemotherapy & $59(52.7)$ & $55(53.9)$ \\
\hline Radiotherapy & $45(40.2)$ & $38(37.3)$ \\
\hline Targeted therapy & $4(3.6)$ & $4(3.9)$ \\
\hline Immunotherapy & $7(6.3)$ & $6(5.9)$ \\
\hline \multicolumn{3}{|c|}{ Receiving interventional bronchoscopy again, n (\%) } \\
\hline Yes & $7(6.3)$ & $7(6.9)$ \\
\hline No & $105(93.8)$ & $95(93.1)$ \\
\hline
\end{tabular}

The "*” indicates patients with more than one surgical site. 

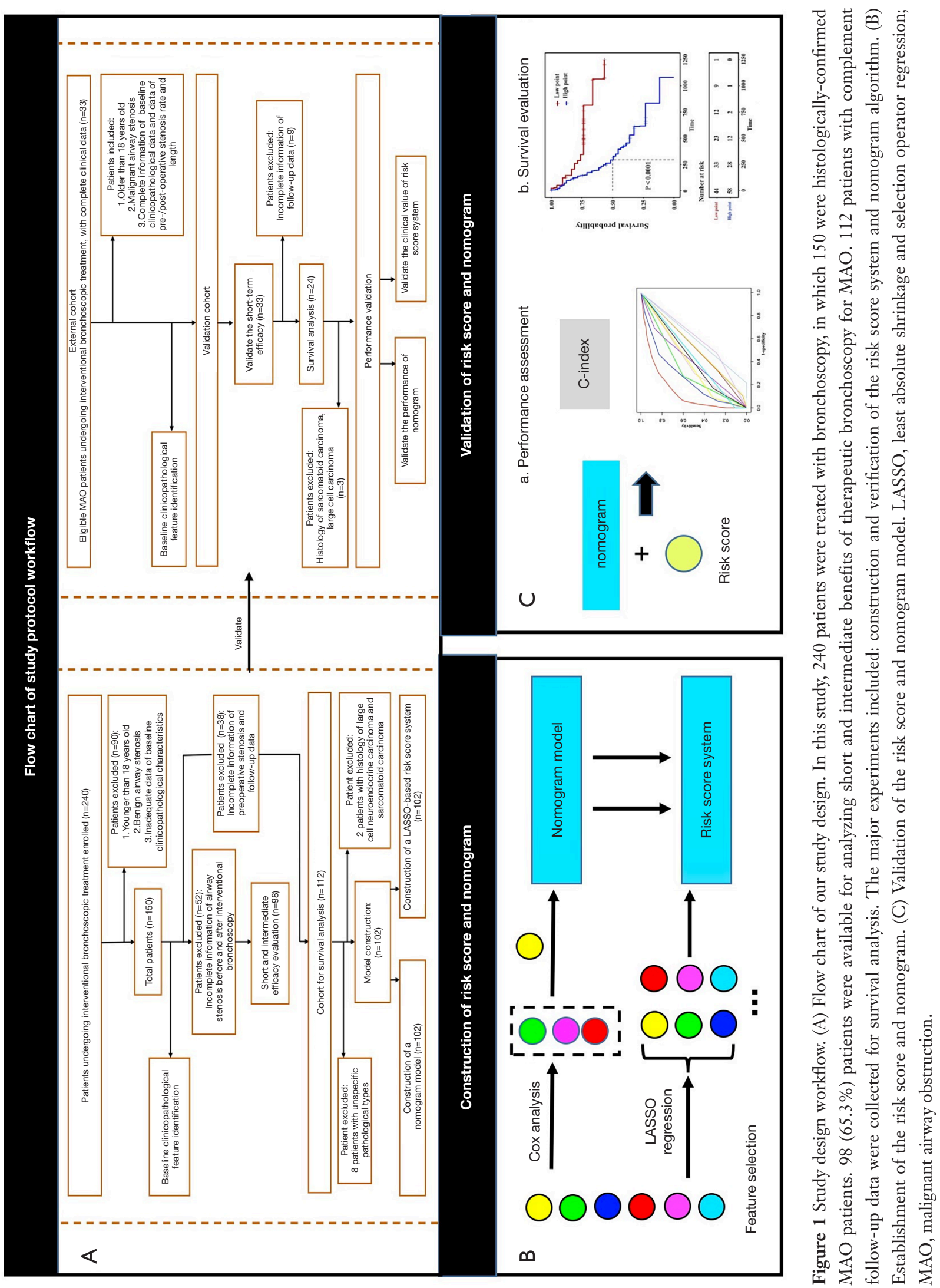

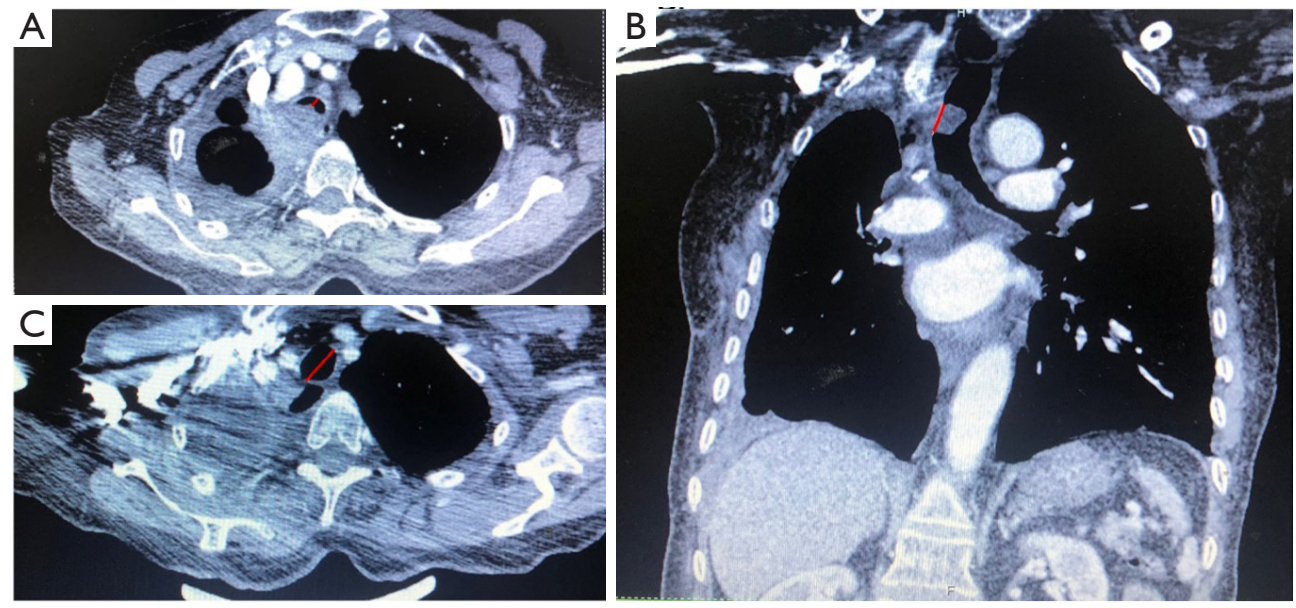

Figure 2 Measurement of the airway stenosis indexes. Lumen diameters were measured in mediastinal window of the dynamic enhanced chest computerized tomography (CT) scans. CT scans of (A) the minimum inner diameter of the stenosis, (B) the stenosis length of the narrow length of the airway, and $(\mathrm{C})$ the inner diameter of the adjacent normal airway. CT, computerized tomography.

\section{Therapeutic bronchoscopy for MAO in our study}

Interventional therapies were performed by the experienced bronchoscopy specialists (YG, DMY, and LY), which included electrocoagulation ( $40 \mathrm{~W})$, stenting, treatment of electrocoagulation plus stenting, treatment of electrocoagulation plus electrosurgical snare, treatment of electrocoagulation, electrosurgical snare, plus stenting, and laser $(20 \mathrm{~W})$ in this study. High-frequency electrosurgical equipment (Tübingen, Germany), metal stent (Nedick, Massachusetts, USA), and semiconductor laser system (Montgomeryville, Pennsylvania, USA) were used. The selection criteria of the interventional bronchoscopy were best determined by stenosis types (25): ablation such as electrocoagulation, electrosurgical snare, and laser were utilized for simple intraluminal malignancies; stents could be placed for simple external stenosis; treatment of ablation plus stent implantation was used for mixed stenosis (25). Specifically, we mainly used laser for tumors causing central airway stenosis and patients with severe shortness of breath; and used electrosurgical snare for tumors with narrow bases. The use of electrocoagulation is relatively wide; however, it was not appropriate for tumors with extremely hard texture, for which laser was the suitable ones. The use of combined therapies was individualized, considering the characteristics of the tumor, such as morphology, compression, and texture, to better treat patients and efficiently relieve symptoms. The specific methods should be determined according to the actual situation of patients. General anesthesia was applied for interventional bronchoscopy and flexible bronchoscopy was used for the procedure.

\section{Imaging analysis}

Lumen diameters were measured in the mediastinal window of the dynamic enhanced chest CT scans. Measurement of the airway diameter included two parts. First, we measured the minimum lumen diameter of stenosis. We then measured the nearest proximal portion of normal diameter, paralleling to the minimum lumen diameter of stenosis. If the top of the trachea was invaded by tumor, we measured the nearest distal portion of normal diameter. When measuring the stenosis length, we manually marked the origin and end of the stenosis in the coronal position of the CT scans (Figure 2). The stenosis rate was calculated as (1the minimum lumen diameter/the nearest distal or proximal portion of normal diameter) $\times 100 \%(26)$.

Stenosis rate $=(1-d 1 / d 2) \times 100 \%$

The $\mathrm{d} 1$ was defined as the minimum lumen diameter of the stenosis; $\mathrm{d} 2$ was defined as the nearest distal or proximal portion of normal diameter.

\section{Determination of imaging cutoff for stenosis rate and length}

We defined the high result as more than $88.46 \%$ for preoperative rate or more than 3.91 centimeters for stenosis length. Best cutoff values were determined using the $\mathrm{R}$ survminer package. 


\section{Nomogram construction and internal verifications}

We randomly divided the whole dataset with 102 patients into training and testing cohorts by $\mathrm{R}$ programming $(7: 3)$. Parameters selected from Cox regression and clinical stage were chosen as factors. Nomogram model was constructed by using "rms" $\mathrm{R}$ package. The performance of this nomogram was measured using the concordance index (C-index) and time-dependent receiver operating characteristic (ROC) curve analyses. Further, Kaplan-Meier survival analyses were performed to evaluate the clinical value of this nomogram model (Figure 1).

\section{Construction of the risk-score system}

Unlike stepwise regression, method of least absolute shrinkage and selection operator (LASSO) regression is able to select independent factors by processing all variables $(27,28)$, which increases model stability dramatically. Clinical factors that were most relevant to survival of MAO patients were screened by using package "glmnet" in R. We also used "cv.glmnet" function for cross validation, avoiding overfitting, and constructed regression models incorporating selected parameters using Cox regression method. To develop a risk score system for $\mathrm{MAO}$ patients, we incorporated the factors selected from model with the optimal performance for Cox multivariate analysis. Risk score for these MAO patients was calculated: Risk score $=\left(0.421^{*}\right.$ age $)-\left(0.039^{*} \mathrm{sex}\right)$ $+\left(0.645^{*}\right.$ smoking status $)+\left(0.076^{*}\right.$ clinical stage $)-$ $\left(0.413^{*}\right.$ receiving interventional bronchoscopy again due to restenosis $)-\left(0.213^{*}\right.$ intraluminal subtype $)+(0.427 *$ external compression subtype $)-\left(1.464^{*}\right.$ histology $)+\left(0.476^{*}\right.$ stenosis rate $)+\left(0.817^{*}\right.$ stenosis length $)-\left(0.504^{*}\right.$ radiotherapy after interventional bronchoscopy $)+\left(0.143^{*}\right.$ immunotherapy after interventional bronchoscopy $)-\left(1.884^{*}\right.$ targeted therapy after interventional bronchoscopy). Furthermore, we proved its risk-discrimination capability among MAO patients and compared it with the single variables and nomogram model (Figure 1).

\section{External validation of the nomogram model}

When using an external cohort for nomogram validation, we calculated the total points for each participant based on the established model. Then, we set the factor by the total points and conducted Kaplan-Meier survival analysis. In addition, C-index as well as ROC curves were derived according to the analysis in this external cohort (29).

\section{Statistical analysis}

Variables with statistical significance $(\mathrm{P}<0.05)$ in the univariate analysis were chosen entering into the multivariate model. Considering multiple clinical parameters, logistic regression analysis was conducted for predicting short and intermediate benefits. We also used Kaplan-Meier analyses and Cox regression for comparing prognosis conditions among diverse populations. For multivariate Cox regression, factors with $\mathrm{P}$ value $<0.1$ in the univariate Cox analysis and clinical stage were included for analysis. OS was determined as the end point. All models used in our study met the prerequisites. We used the area under the receiver-operating characteristic curve (AUC) to evaluate the discriminative capability of the models. Data analysis and visualization were performed using $R$ version 3.6.1 (R Foundation for Statistical Computing), GraphPad Prism version 7 for Mac OS X (GraphPad Software, San Diego, California), Excel (Excel for Mac 2016, Microsoft, Redmond, Washington), and SPSS statistical software version 26.0 (IBM Corp., Armonk, New York). A two-tailed $\mathrm{P}<0.05$ was defined as statistical significance.

\section{Results}

\section{Clinicopathological characteristics}

In our study, patients are predominantly male $(81.3 \%)$ and the median age was 62.0 years (Table S1). 147 (98.0\%) patients had clinical advanced-stage (III-IV) malignant disease and most tumors were SCC and ADC (73.3\%). For the dataset to study survival, most were males, with a mean age of $61.7 \pm 9.9$ years. $98.2 \%$ patients were at advanced stages (stage III-IV) and SCC was the most common histologic subtype (Table 1). Minimal bleeding was observed during the procedures among these patients. $6.3 \%$ patients $(7 / 112)$ had restenosis and all of these patients with restenosis received interventional bronchoscopy again (Table 1).

\section{Short and intermediate benefits}

To investigate the short (within 30 days) and intermediate efficacy (more than 30 days) among $\mathrm{MAO}$ patients undergoing interventional bronchoscopy, we identified and compared the stenosis rate of each patient before and after the therapy. In all 98 patients, we observed a significant decline of airway stenosis after bronchoscopy from $71.7 \% \pm 2.1 \%$ to $36.6 \% \pm 2.7 \%(\mathrm{P}<0.001)$ (Figure $3 A, B, C, D, E)$. Among them, 
67 (67/98, 68.4\%) showed short or intermediate benefits (the postoperative lumen reopened more than $50 \%$ of the normal), which indicated that bronchoscopic therapy could dilate patient's airway effectively. Considering the diversely clinical classifications of bronchoscopic therapies, we further surveyed the therapeutic efficacy of each interventional program. It is worth noting that stenosis rate before receiving treatment of electrocoagulation ( $v s$. after electrocoagulation therapy $70.3 \% \pm 3.3 \%$ vs. $40.7 \% \pm 3.8 \%, \mathrm{P}<0.001)$, stenosis rate before receiving treatment of stenting ( $v s$. after stenting therapy $68.9 \% \pm 4.3 \%$ vs. $25.6 \% \pm 4.7 \%, \mathrm{P}<0.001)$, stenosis rate before receiving treatment of electrocoagulation in combination with electrosurgical snare (vs. after combined therapy $79.1 \% \pm 3.5 \%$ vs. $44.5 \% \pm 7.4 \%, \mathrm{P}<0.001)$, stenosis rate before receiving treatment of electrocoagulation in combination with stenting (vs. after combined therapy $79.2 \% \pm 5.3 \%$ vs. $34.3 \% \pm 9.0 \%$, $\mathrm{P}<0.001)$ marked declined, echoing the conception that interventional bronchoscopic therapy was very useful to relieve symptoms (Figure 3B,C,D,E). Among different operations, electrocoagulation in combination with airway stent implantation showed the maximal decline of stenosis rate $(44.82 \%$ before and after therapy) (Figure $3 E$ ). Thus, interventional bronchoscopy played an important role in improving the short- and intermediate conditions of patients with MAO. In addition, we also identified the roles of clinical characteristics in predicting short and intermediate benefits of MAO. However, we failed to find determinants of the short and intermediate outcomes ( $\mathrm{P}>0.05$; Table $\mathrm{S} 3)$.

For short-term efficacy, in all $38 \mathrm{MAO}$ patients, similar results were showed (Figure S1). Intermediate efficacy was also observed with significant stenosis improvement in 60 MAO patients (Figure S2). With continuous follow-up (from the first-time operation to death or deadline for follow-up), lumen restenosis was observed in 6 of these $98(6 / 98,6.1 \%)$ $\mathrm{MAO}$ patients (Figure S3).

\section{Prognosis of MAO patients after interventional bronchoscopy}

We obtained 112 samples with complete survival data (Figure $3 F$ ), with a median survival time of 614.000 days (95\% CI: 269.876-958.124; Figure 3G). In all $66 \mathrm{MAO}$ patients less than 65 years old, $25(37.9 \%)$ reached the end event for OS (median 1,065.000 days, 95\% CI: undefined). For 46 patients more than 65 years old, the median OS was 288.000 days (95\% CI: 121.514-454.486), among them $16(16 / 46,34.8 \%)$ had survived. Kaplan-Meier analysis showed that the basic characteristics including age
( $\mathrm{P}=0.004)$, smoking status $(\mathrm{P}=0.005)$, preoperative stenosis rate $(\mathrm{P}=0.006)$ were significantly associated with MAO OS (Table S4). Meanwhile, stenosis site $(\mathrm{P}=0.056)$ and airway stenosis classification $(\mathrm{P}=0.051)$ were analyzed as marginally significant factors for MAO OS. There was no statistical difference in survival observed in clinical stage II-III and IV $(\mathrm{P}=0.465) .102$ patients were available for studying the impacts of histology on patients' prognosis. As Figure $3 \mathrm{H}$ showed, adenoid cystic carcinoma had better prognosis than SCC $(\mathrm{P}=0.002)$, ADC $(\mathrm{P}=0.008)$, and SCLC $(\mathrm{P}=0.006)$.

Then, we evaluated the prognosis of MAO patients undergoing different interventional methods (Figure 3I, f, K,L, M). Among all therapeutic bronchoscopies except electrosurgical snare $(\mathrm{n}=1)$, patients receiving treatment of electrocoagulation plus electrosurgical snare showed the longest survival, followed by receiving electrocoagulation alone, receiving stenting alone, and combining electrocoagulation and stenting (Figure $3 M$, $\mathrm{P}=0.022$ ). Four patients received laser treatment and have maintained good condition after surgery, with a period of 494, 448, 474, and 663 days respectively since the operation. The OS of two patients receiving the combined therapy of electrocoagulation, electrosurgical snare and stenting was lower than the median survival (147 and 55 days respectively).

\section{COX regression for $O S$ in $M A O$}

To excavate the potential factors influencing the survival, we used patients' baseline clinicopathological features for regression analysis (Table 2). A total of $102 \mathrm{MAO}$ patients with known histological diagnosis were enrolled for the study. Univariate analysis revealed age $<65$ years $(\mathrm{P}=0.007)$, nonsmoking status $(\mathrm{P}=0.004)$, adenoid cystic carcinoma $(\mathrm{P}=0.016)$, and low stenosis rate $(\mathrm{P}=0.007)$, as being associated with better OS significantly. Intriguingly, multivariate analysis identified nonsmoking status, adenoid cystic carcinoma, and low preoperative stenosis length as independent predictive factors for better OS of MAO patients [vs. smokers, HR=0.425, 95\% CI: 0.228-0.794, $\mathrm{P}=0.007$; vs. non-adenoid cystic carcinoma (SCC, ADC, and SCLC), HR=0.100, 95\% CI: 0.011-0.898, $\mathrm{P}=0.040$; high preoperative stenosis length, $\mathrm{HR}=0.409$, 95\% CI: $0.207-$ $0.811, \mathrm{P}=0.011]$.

\section{Nomogram model for prognostic prediction}

Given the significant results of Cox regression and the 

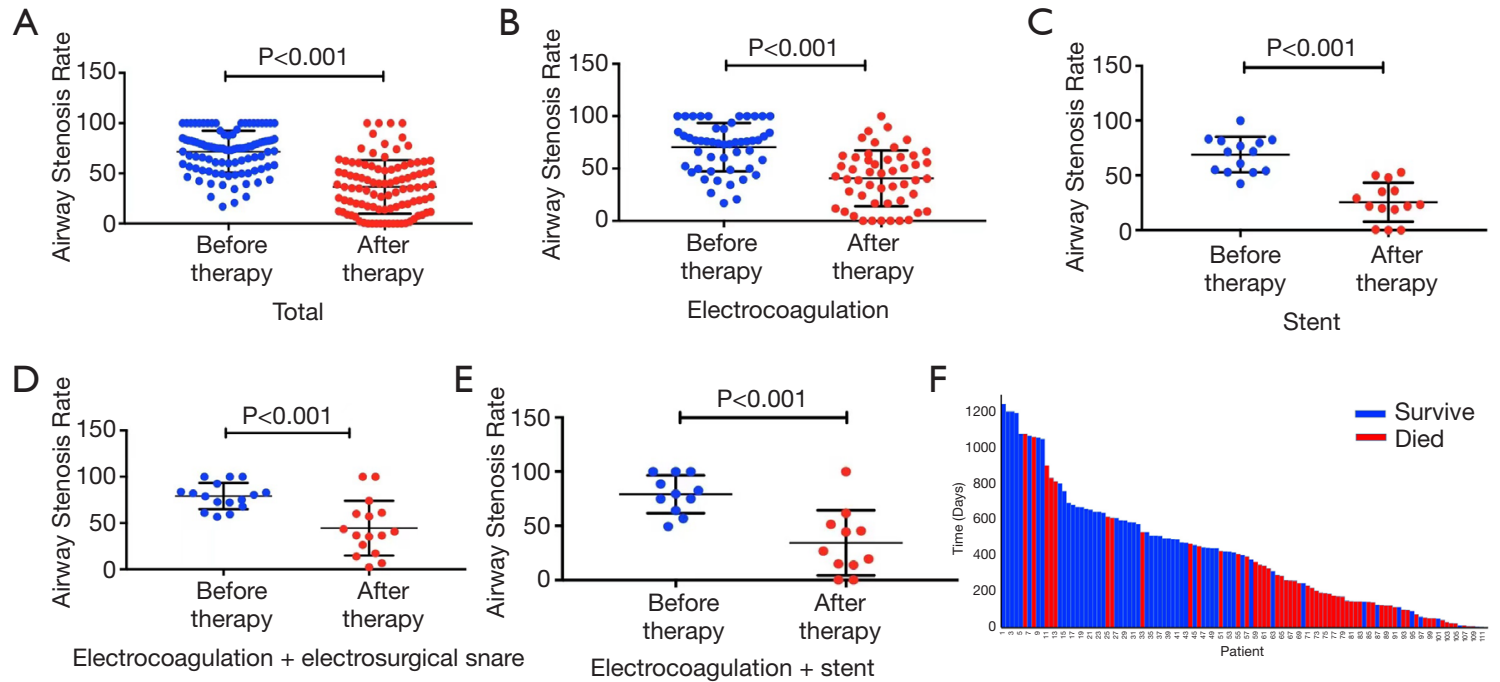

G
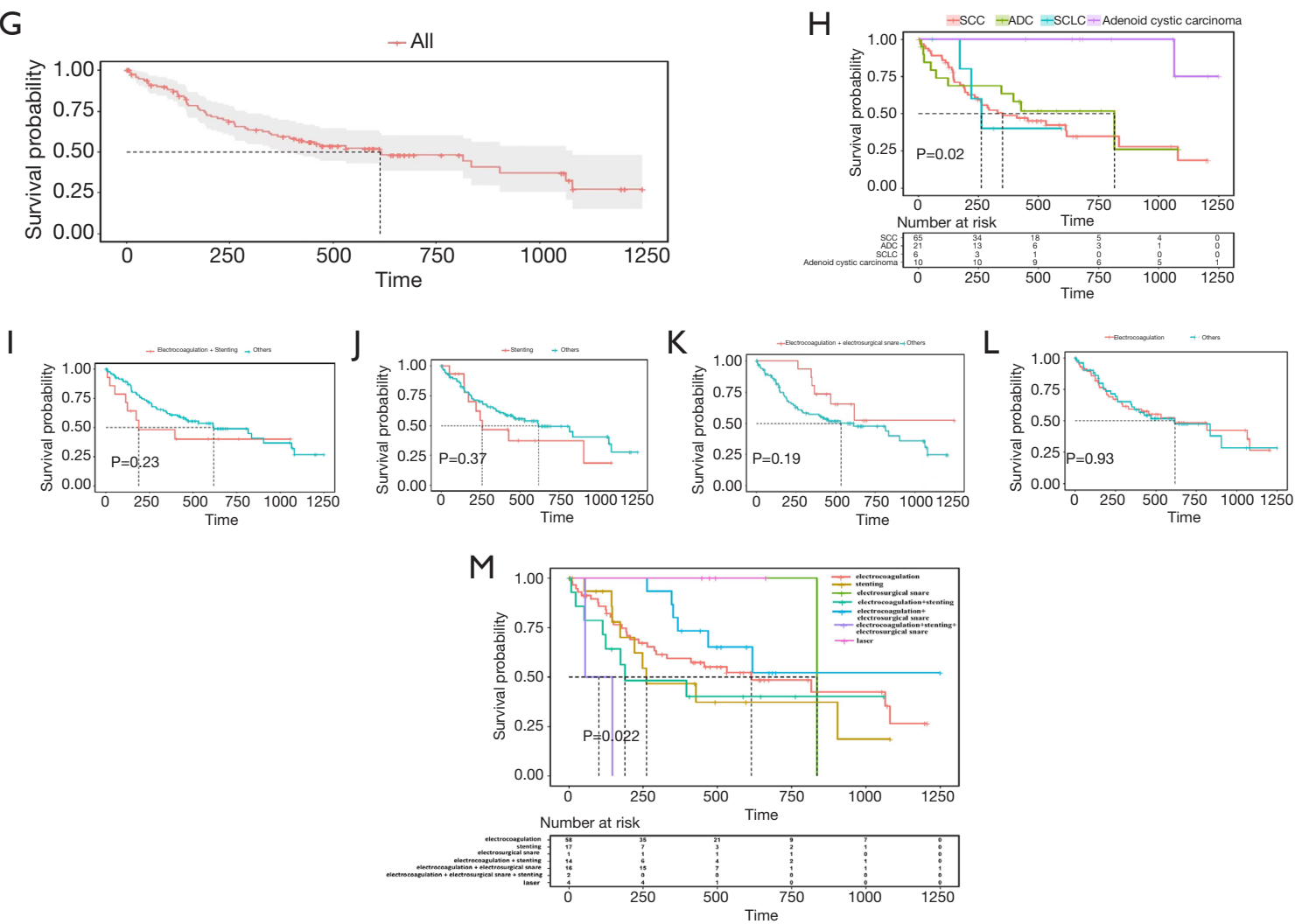

Figure 3 Airway stenosis rate before and after treatment and survival of MAO patients undergoing interventional bronchoscopy. Stenosis rate changes with interventional bronchoscopic therapies including (A) all treatments involved (N=98), (B) electrocoagulation alone $(\mathrm{N}=50)$, (C) stenting alone $(\mathrm{N}=14),(\mathrm{D})$ combining therapy of electrocoagulation and electrosurgical snare $(\mathrm{N}=16),(\mathrm{E})$ combining therapy of electrocoagulation and stenting (N=11), (F) Survival status and survival period. 112 patients with complete survival data were enrolled for survival study. (G) The Kaplan-Meier survival curves of 112 patients. (H) Kaplan-Meier survival analysis of MAO patients based on histological subtypes ( $\mathrm{n}=102)$. (I,J,K,L) The Kaplan-Meier survival curves comparing prognosis of MAO patients receiving electrocoagulation plus stenting, stenting implantation, electrocoagulation plus electrosurgical snare, or electrocoagulation, with others respectively. (M) The Kaplan-Meier survival analyses of distinct therapeutic bronchoscopic methods. MAO, malignant airway obstruction; SCC, squamous cell carcinoma; ADC, adenocarcinoma; SCLC, small-cell lung cancer. 
Table 2 Univariate and multivariate analysis of MAO patients undergoing interventional bronchoscopy

\begin{tabular}{|c|c|c|c|c|c|c|c|c|}
\hline Factor & \multicolumn{2}{|c|}{ No. of patients } & \multicolumn{3}{|c|}{ Univariate analysis } & \multicolumn{3}{|c|}{ Multivariate analysis } \\
\hline Sex (female vs. male) & $17,16.7$ & $85,83.3$ & 0.541 & $0.230-1.273$ & 0.159 & & & \\
\hline Age (<65 vs. $\geq 65)$ & $61,59.8$ & $41,40.2$ & 0.468 & $0.271-0.809$ & 0.007 & 0.580 & $0.319-1.055$ & 0.074 \\
\hline $\begin{array}{l}\text { Smoking (nonsmoker vs. } \\
\text { smoker) }\end{array}$ & $56,54.9$ & $46,45.1$ & 0.435 & $0.246-0.770$ & 0.004 & 0.425 & $0.228-0.794$ & 0.007 \\
\hline $\begin{array}{l}\text { Lesion and surgical site } \\
\text { (Trachea and carina vs. } \\
\text { bronchus) }\end{array}$ & $41,40.2$ & $61,59.8$ & 0.638 & $0.355-1.146$ & 0.133 & & & \\
\hline Stenosis subtypes & & & & & 0.080 & & & 0.232 \\
\hline $\begin{array}{l}\text { Stenosis subtypes } \\
\text { (external compression } \\
\text { vs. mixed type) }\end{array}$ & $8,7.8$ & $72,70.6$ & 1.581 & $0.617-4.051$ & 0.340 & 2.152 & $0.785-5.904$ & 0.137 \\
\hline $\begin{array}{l}\text { *Histology (non-adenoid } \\
\text { cystic carcinoma vs. } \\
\text { adenoid cystic carcinoma) }\end{array}$ & $92,90.2$ & $10,9.8$ & 11.592 & $1.583-84.889$ & 0.016 & 9.994 & $1.113-89.698$ & 0.040 \\
\hline Stenosis rate (low vs. high) & $81,79.4$ & $21,20.6$ & 0.435 & $0.237-0.798$ & 0.007 & 0.639 & $0.325-1.254$ & 0.193 \\
\hline $\begin{array}{l}\text { Stenosis length (low vs. } \\
\text { high) }\end{array}$ & $77,75.5$ & $25,24.5$ & 0.598 & $0.326-1.097$ & 0.097 & 0.409 & $0.207-0.811$ & 0.011 \\
\hline $\begin{array}{l}\text { Targeted therapy } \\
\text { after interventional } \\
\text { bronchoscopy (no vs. yes) }\end{array}$ & $98,96.1$ & $4,3.9$ & 21.463 & $0.031-14997.714$ & 0.359 & & & \\
\hline $\begin{array}{l}\text { Immunotherapy } \\
\text { after interventional } \\
\text { bronchoscopy (no vs. yes) }\end{array}$ & $96,94.1$ & $6,5.9$ & 0.603 & $0.216-1.680$ & 0.333 & & & \\
\hline $\begin{array}{l}\text { Receiving interventional } \\
\text { bronchoscopy again (no } \\
\text { vs. yes) }\end{array}$ & $95,93.1$ & $7,6.9$ & 1.643 & $0.506-5.334$ & 0.409 & & & \\
\hline
\end{tabular}

HR, hazard ratio; MAO, malignant airway obstruction; $95 \% \mathrm{Cl}, 95 \%$ confidence interval. *, non-adenoid cystic carcinoma: squamous cell carcinoma, adenocarcinoma, small cell lung cancer. 
important value of clinical stage, we incorporated smoking status, preoperative stenosis length, histologic subtype, and clinical stage, to establish the quantitative means for survival prediction of MAO (Figure 4A). Then, we used C-index for evaluating its predictive value, and the nomogram system performed well with the C-index of $0.668,0.695$, and 0.669 in the training, testing, and whole MAO cohorts, respectively. Further, 1-, 2-, and 3-year time-dependent ROC curves also indicated excellently predictive ability (AUC $0.651,0.783$, and 0.739 for the training cohort respectively; for the testing cohort, all of the AUC values were 0.738 ; Figure $4 B, C$ ).

Moreover, based on the median value of the total points calculated from this nomogram, we further divided these $102 \mathrm{MAO}$ patients into higher score and lower score groups. Patients with lower score exhibited the higher survival rate compared with those with higher score (for the entire cohort, third quantile of OS: low score $v s$. high score, 346.000 days, $95 \%$ CI: 0.000-902.050 vs. 144.000 days, 95\% CI: 94.185-193.815; $\mathrm{P}<0.001$; for training cohort, third quantile of OS, low score vs. high score, 816.000 days, $95 \%$ CI: $0.000-1,811.619$ vs. 150.000 days, $95 \%$ CI: 35.166-264.834; $\mathrm{P}=0.001$; for testing cohort, third quantile of OS: low score $v s$. high score, 261.000 days, $95 \%$ CI: 19.920-502.080 vs. 126.000 days, $95 \%$ CI: 74.419-177.581; $\mathrm{P}=0.050$; Figure $4 D, E, F)$.

\section{Construction of risk-score system based on LASSO regression}

Considering widespread relationships of clinical parameters with MAO prognosis, we proposed to perform LASSO regression for feature selection and construct a fusion biomarker for prognostic evaluation of MAO. The optimal model selected by LASSO was constructed incorporating 13 indicators including age, sex, smoking status, clinical stage, intraluminal subtype, external compression subtype, histology, preoperative stenosis rate, preoperative stenosis length, radiotherapy after interventional bronchoscopy, immunotherapy after interventional bronchoscopy, targeted therapy after interventional bronchoscopy, as well as receiving interventional bronchoscopy again (Figure $5 A, B$ ). Thus, these 13 indicators were chosen for risk-score construction. This risk score achieved excellent performance, with AUC values of $0.785,0.855$, and 0.880 for 1, 2, and 3-year time-dependent ROC curves. Compared with single indicator, this risk score demonstrated better performance. Time-dependent ROC analyses indicated the risk-score with the maximum AUC value of 0.855 , while $0.662,0.598,0.574,0.476,0.466,0.596,0.392,0.615$, $0.573,0.403,0.555$, and 0.459 for single parameters smoking status, age, gender, clinical stage, receiving interventional bronchoscopy again, stenosis classification, histology, preoperative stenosis rate, preoperative stenosis length, radiotherapy or immunotherapy or targeted therapy after interventional bronchoscopy when selecting cutoff value as two years (Figure 5C). Considering the good performance of nomogram, we also compared this risk score system with nomogram. Time-dependent ROC curve showed that the AUC values were 0.855 and 0.739 respectively (Figure 5C), which indicated that this risk score system performed better than the nomogram model to predict prognosis of MAO patients. Using the median value of risk score for risk classification, survival curves showed that high risk-score group had poorer prognosis than low riskscore group (third quantile of OS: 126.000 days, 95\% CI: 73.588-178.412 vs. 532.000 days, $95 \%$ CI: $0.000-1,110.372$; $\mathrm{P}<0.001$; Figure 5D). Univariate Cox regression also analyzed the significant difference between this score and MAO survival (low score $v$ s. high score HR $=0.181,95 \%$ CI: 0.093-0.351; $\mathrm{P}<0.001)$.

\section{Validation by external data}

Clinical characteristics of external MAO patients were summarized (Table S5). Complete preoperative and postoperative stenosis data of MAO patients were available for thirty-three samples. For short-term efficacy, 23 patients $(23 / 33,69.7 \%)$ showed airway reopened more than $50 \%$. Significant differences before and after interventional bronchoscopy were observed in the entire MAO validation cohort $(\mathrm{P}<0.001)$, patients with electrocoagulation in combination with stenting $(\mathrm{P}<0.001)$, and patients with single electrocoagulation therapy $(\mathrm{P}=0.001$; Figure $6 A, B, C)$. Among the external dataset, a total of twenty-four patients were available for survival analysis. In the validation cohort for survival analysis, 95.8\% (23/24) patients reached end points, with a median OS of 122.000 days (95\% CI: 15.178-228.822). Survival analysis of MAO patients in the validation participants was summarized in Table S6. We also verified the prognostic value of the risk-score in this external verification cohort. When applying the same methods to define cutoff values of preoperative stenosis rate and length, we found that the 1-, 2-, 3-year time-dependent ROC analyses also validated good performance of this LASSO-based model, with AUC values of $0.800,0.739$, and 0.739 respectively (Figure $6 D$ ). In addition, using the median 


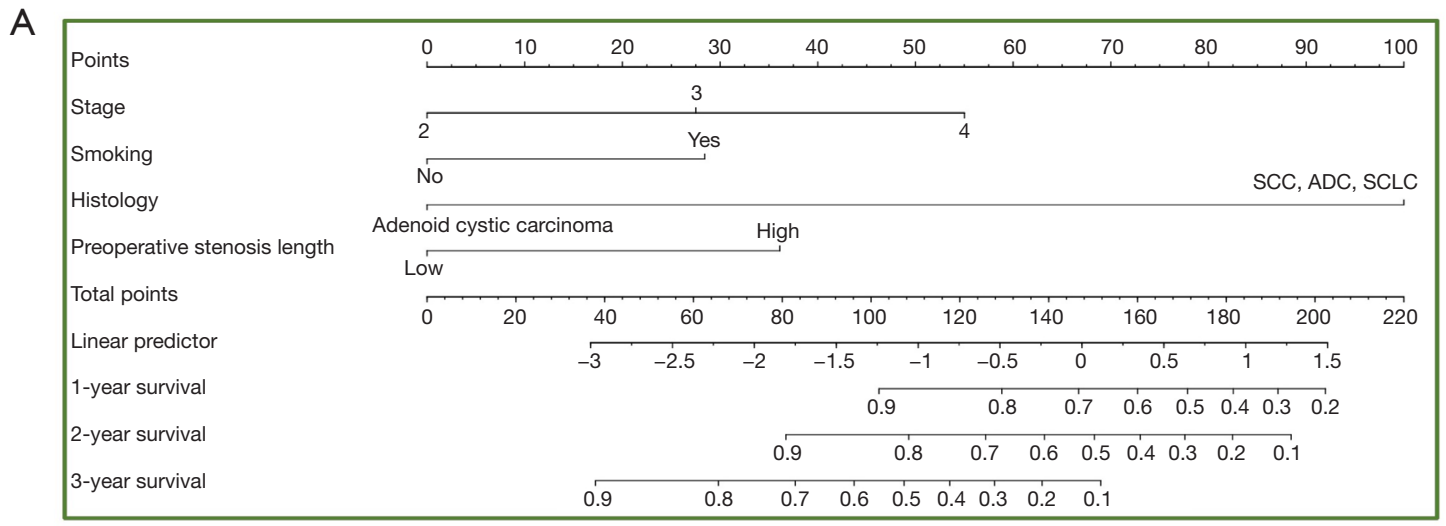
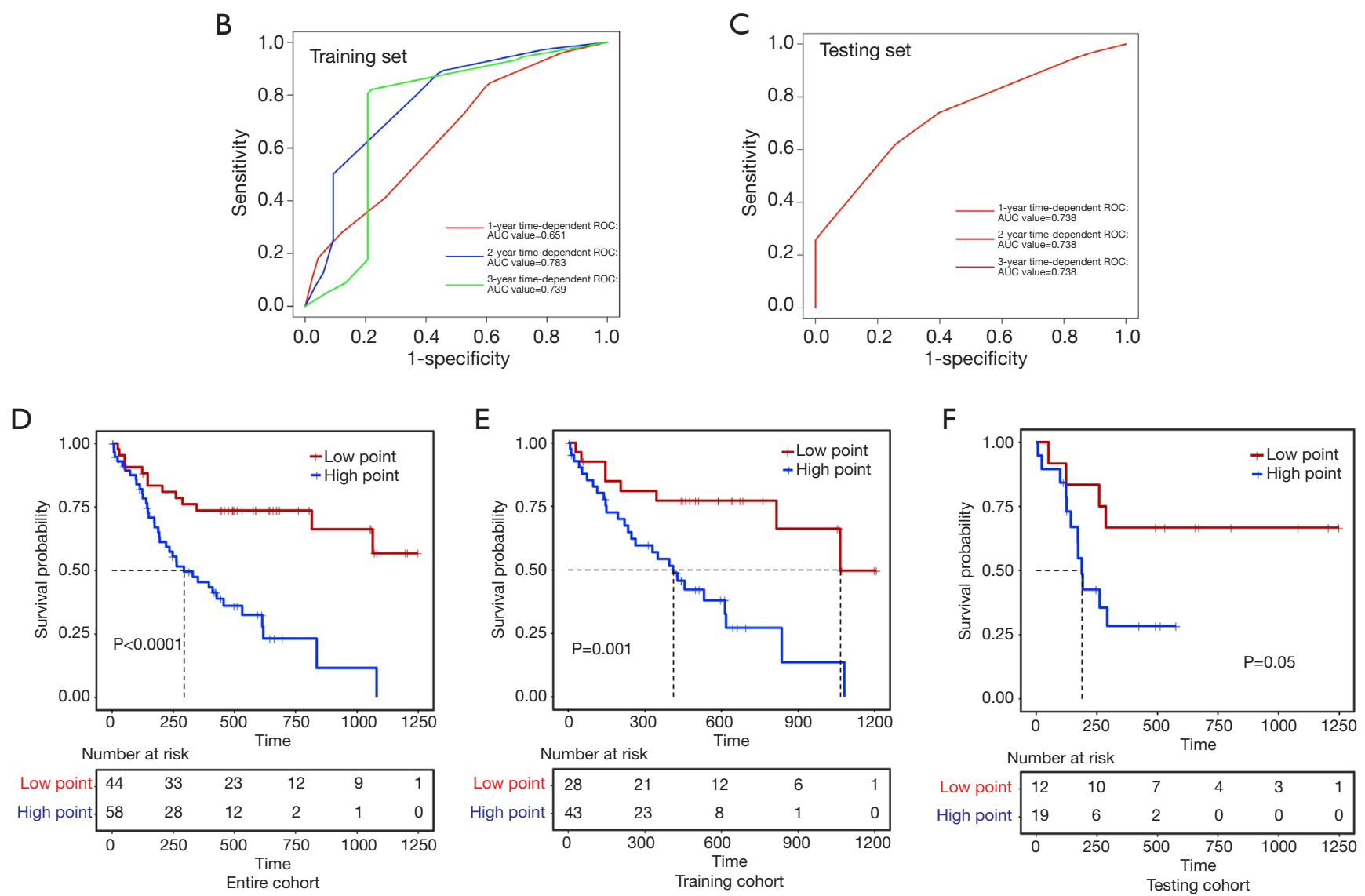

Figure 4 Construction and clinical value evaluation of the prognosis nomogram model for MAO. (A) The nomogram model incorporated preoperative stenosis length, smoking status, clinical stage, and histology. (B,C) 1-, 2-, and 3-year time-dependent ROC curves in the training $(\mathrm{N}=71)$ and testing cohorts $(\mathrm{N}=31)$. (D,E,F) Survival analyses of patients in different total points of the nomogram model in the training, test, and whole MAO cohorts respectively. All three cohorts obtained excellent risk-grade division with significant differences. MAO, malignant airway obstruction; SCC, squamous cell carcinoma; ADC, adenocarcinoma; SCLC, small-cell lung cancer. 

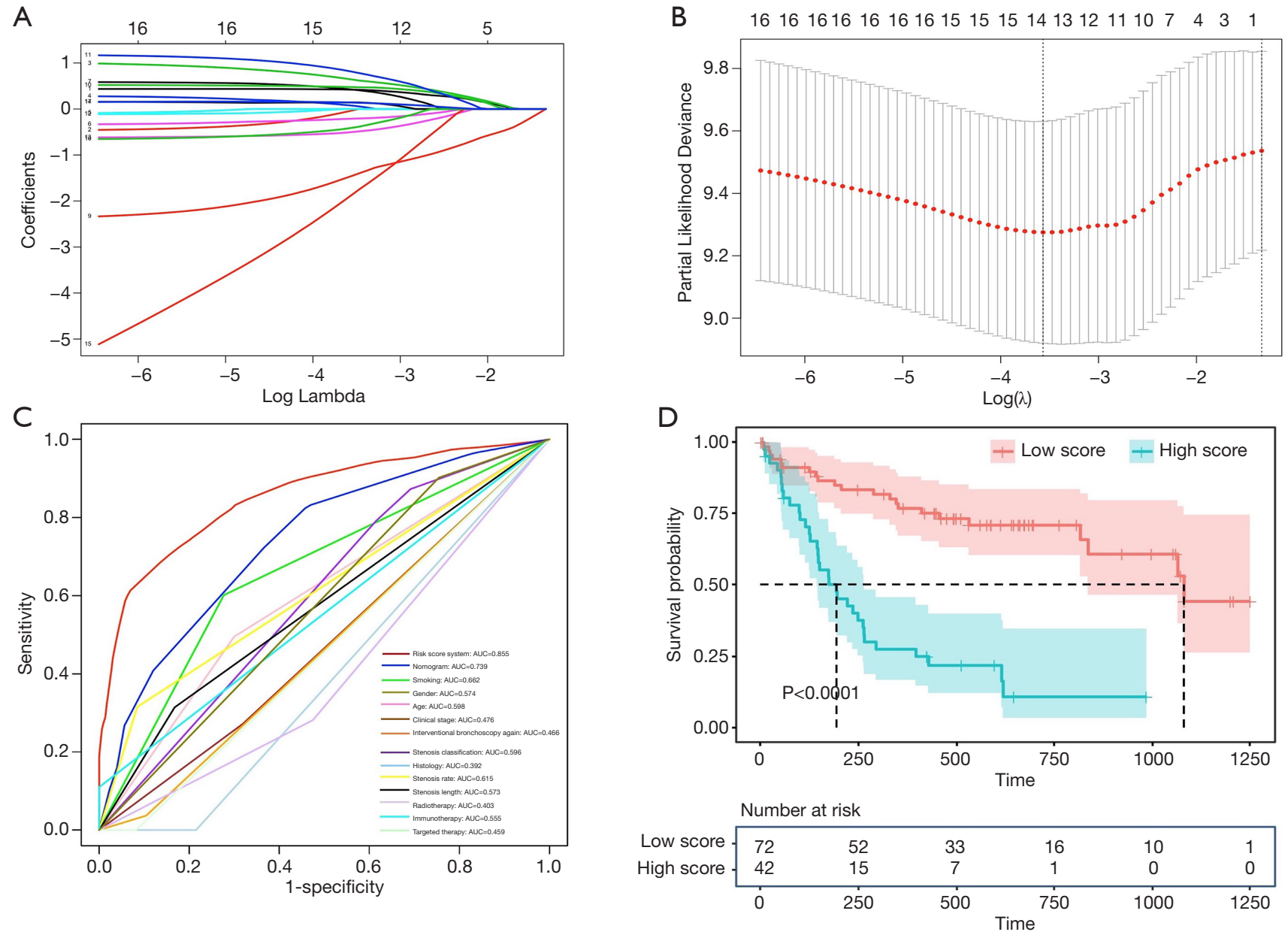

Figure 5 Risk score system and feature importance selection. (A,B) Method of LASSO for feature selection. The results by LASSO selected 13 variates (age, sex, smoking status, clinical stage, intraluminal subtype, external compression subtype, histology, preoperative stenosis rate, preoperative stenosis length, radiotherapy after interventional bronchoscopy, immunotherapy after interventional bronchoscopy, targeted therapy after interventional bronchoscopy, and receiving interventional bronchoscopy again due to restenosis), as predictive factors in all 102 MAO patients to construct the optimal model. (C) Time-dependent ROC analyses for performance evaluation of this predictive riskscore, nomogram, and other single biomarkers. The curves indicated the risk-score with the maximum AUC value of 0.855 , compared with other biomarkers. (D) Survival analysis between high risk-score group and low risk-score group. AUC, area under the receiver-operating characteristic curve; ROC, receiver operating characteristic; LASSO, least absolute shrinkage and selection operator regression; MAO, malignant airway obstruction.

value of risk score for classification, we also found that MAO patients with low risk-score had higher OS (vs. high risk-score group 160.000 days, $95 \%$ CI: 0.000-466.804 vs. 63.000 days, 95\% CI: 26.316-99.684; $\mathrm{P}=0.015$; Figure 6E). Moreover, we also verified the performance and clinical value of this nomogram model (Figure 6F,G). Consistent with our developing cohort, this nomogram model showed relatively poorer performance than LASSO-based risk system, with a C-index of 0.619 as well as AUC values of 1-,
2-, 3-year time-dependent ROC of 0.720, 0.574, and 0.574, respectively. Patients with low nomogram points showed longer OS compared with those with high points (low vs. high, 160.000 days, $95 \%$ CI: 0.000-434.414 vs. 65.000 days, 95\% CI: 0.000-136.634; $\mathrm{P}=0.517)$.

\section{Discussion}

In this study, we first investigated the therapeutic benefits 

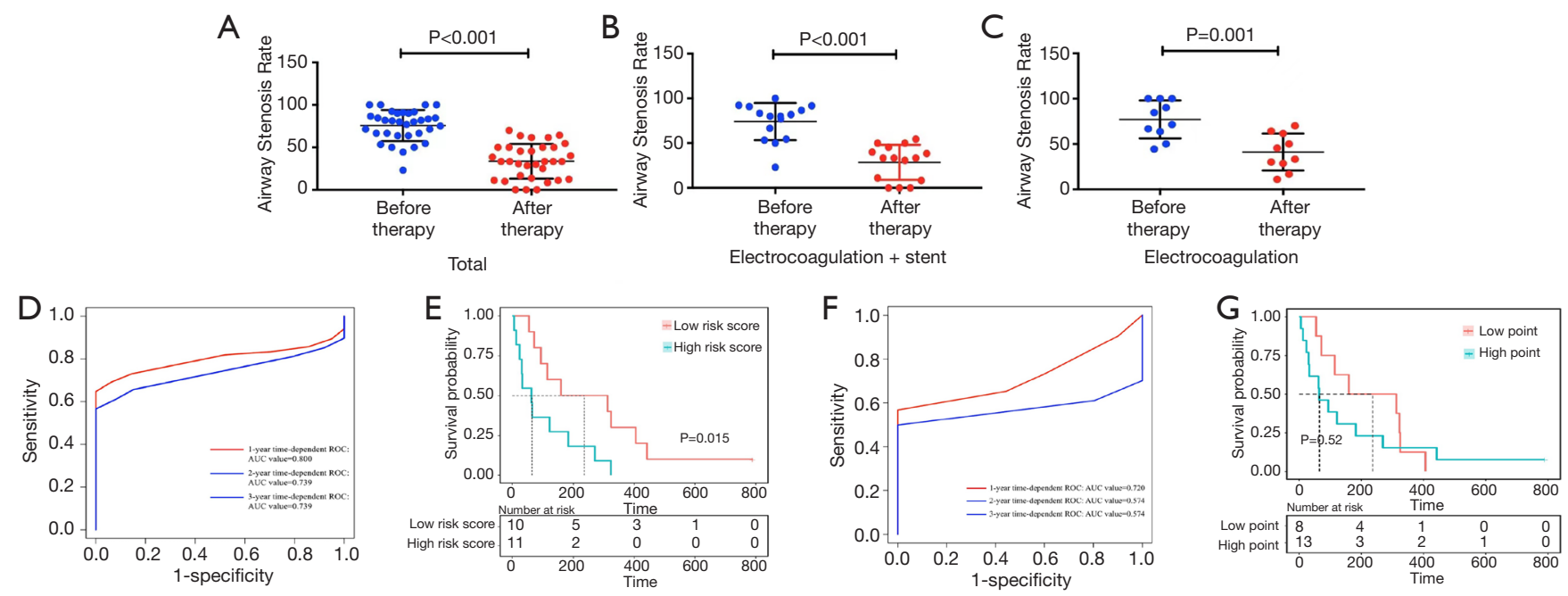

Figure 6 Validation of the risk score and nomogram model on the external data. (A,B,C) Significant differences before and after interventional bronchoscopy were observed in the entire $\mathrm{MAO}$ validation cohort $(\mathrm{P}<0.001)$, patients with electrocoagulation in combination with stenting $(\mathrm{P}<0.001)$, and patients with single electrocoagulation therapy $(\mathrm{P}=0.001)$. (D) Time-dependent ROC for the LASSO-based risk score system. (E) Survival analysis of the risk-score in external dataset. (F) Time-dependent ROC for nomogram. (G) Survival analysis based on nomogram in external dataset. AUC, area under the receiver-operating characteristic curve; LASSO, least absolute shrinkage and selection operator regression; MAO, malignant airway obstruction; ROC, receiver operating characteristic.

of MAO patients undergoing interventional bronchoscopy and explored patients' prognosis of distinct methods of therapeutic bronchoscopy and other clinical characteristics. Then, univariate and multivariate Cox analyses were conducted and a nomogram predictive model based on histology, clinical stage, smoking status, and preoperative stenosis length was constructed for prognosis prediction of MAO. In addition, considering the impacts of different clinical factors on MAO prognosis, we then conducted LASSO algorithm, and established a well-performed risk score system incorporating 13 indicators including age, sex, smoking status, clinical stage, intraluminal subtype, external compression subtype, histology, preoperative stenosis rate, preoperative stenosis length, radiotherapy after interventional bronchoscopy, immunotherapy after interventional bronchoscopy, targeted therapy after interventional bronchoscopy, and receiving interventional bronchoscopy again due to restenosis. Interestingly, this risk score obtained a better AUC value than the above nomogram model and single indicators. We also verified the stenosis characteristics before and after bronchoscopy, as well as the clinical values of the nomogram model and risk score system in an external dataset, which further proved the good performance of LASSO-based risk score system. More prospective trials should be carried out for verification.

As the results shown in our study, interventional therapy expanded airway successfully, which was similar to previous research results $(2,30,31)$. Some previous studies also illustrated improvement in dyspnea and life quality after bronchoscopy (5,32-34). Airway restenosis was observed in 9 patients among all 150 MAO patients enrolled. The time from the first interventional bronchoscopy to receiving interventional bronchoscopy again due to restenosis in these patients ranged from 16 to 709 days. This restenosis could relate to tumor ingrowth or excessive mucus retention and granulation tissue $(32,35-37)$. The restenosis of seven of the nine patients was caused by tumor ingrowth and two was caused by granulation tissue. Interventional pulmonology procedures were re-performed for all of these patients with restenosis. No severe complications were encountered.

Patients chosen for interventional bronchoscopy were mainly at advanced stages in our study. They suffered from airway stenosis in different degrees and some of them experienced symptoms such as chest tightness and shortness of breath. The main purpose of interventional bronchoscopy for these advanced patients was to remove blockage of airway, relieve symptoms, and prolong life to some extent. A total of 3 patients at stage II received interventional bronchoscopy in all 150 patients. Patient 1 had a severe 
reduction of pulmonary ventilation, with the forced expired volume in one second as a percentage of expected value (FEV1\% Pred) of $37.2 \%$, combined with pulmonary bullae. For patient 2 and 3, during bronchoscopy, new organisms in the left lower lobar and right lower lobar bronchi were found respectively, which greatly clogged the tube cavity. Therefore, interventional bronchoscopy was recommended. The treatment of interventional bronchoscopy could help to keep the airway patency, assess the extent of tumor invasion, and provide guidance for resection range of surgery to some extent. The basic purpose of intervention for patients at early and advanced stages was to remove blockages and ensure airway patency. The median survival time was 614 days in this study, which was comparatively longer than the results of other published reports $(2,15,38)$. Armin Ernst and colleagues showed a 30-day mortality of $7.8 \%$ in $\mathrm{MAO}$ patients after bronchoscopy, with common complications occurring, associated with intervention urgency and health status (39). Many studies also indicated that timely therapeutic bronchoscopy could improve patients' life quality and survival $(34,40,41)$. We also evaluated patients' survival after distinct interventional bronchoscopies and found different clinical outcomes among them. They suffered from different degrees of stenosis by the tumor. Thus, it is important to explore the potential factors that might affect patients' survival.

In our study, we found that age $<65$ years old, nonsmoking status, adenoid cystic carcinoma, and low preoperative stenosis rate, were significantly associated with longer $\mathrm{OS}$ in $\mathrm{MAO}$ patients $(\mathrm{P}<0.05)$. After multivariate Cox regression incorporating more factors, the independently prognostic values of smoking status, histology, and preoperative stenosis length were revealed. Compared with endobronchial and mixed tumor types, patients with extrinsic tumors showed shorter OS; lesions in trachea and carina tended to have better clinical outcomes than bronchus lesions, although only marginally significant difference was revealed. Patients experienced respiratory failure before interventional bronchoscopy showed shorter OS compared with those with no respiratory failure. After bronchoscopy and intensive treatment, one patient survived for 396 days, and the other was still alive and having already survived for 513 days. However, only two patients with respiratory failure were included. For a better understanding of the prognosis of MAO patients with respiratory failure before bronchoscopy, a larger prospective study is needed. No MAO patient caused by esophageal cancer was enrolled for study, and more studies were needed to explore the prognosis of MAO patients caused by tumors such as esophageal cancer. Previous study also suggested functional status, cardiac disease, dyspnea level, time from disease diagnosis to interventional bronchoscopy, bronchoscopic dilation and chemotherapy were correlated with MAO patients' prognosis (18). Thus, many factors should be considered when evaluating prognosis of MAO patients after interventional bronchoscopy.

Nomogram is increasingly developed as a promising method for predicting patients' prognosis, and several models could perform better than the traditional clinical systems $(29,42,43)$. Thus, based on survival analysis, we first constructed a nomogram incorporating histology, clinical stage, smoking status, and preoperative stenosis length for prediction. In addition, considering multiple parameters could influence patients' survival, we further conducted LASSO regression and established a riskscore system with good performance to predict prognosis. Both predictive systems showed patients with low score exhibited higher survival rate as potential biomarkers. Thus, we then conducted time-dependent ROC curve analysis, given the well-performance of both models, to compare their performance. The risk-score system was proved better performed. Also, when compared with single biomarkers, this risk-score system exhibited the best performance. The AUC values were 0.855 and 0.476 for risk score system and staging, which indicated that this risk score might represent a promising method for prognosis evaluation when compared with the conventional stage system among these patients. It could predict patients' long-term prognosis receiving bronchoscopy better than the current stage system. Unlike single biomarkers, the risk score system combined both traditional staging system and other clinical parameters to construct a fusion biomarker, which is of great importance for the individualization of prognostic evaluation of MAO patients with interventional therapy.

To further understand patients' survival characteristics and evaluate clinical values of two predictive systems, we collected relevant data in another institution as an external dataset. Consistent with our data, in the external cohort, we observed significant differences before and after interventional bronchoscopy. When verifying the prognostic value of the LASSO-based risk-score in external dataset, we proved its outstanding performance and verified significantly longer survival in patients with low-risk score, which further proved the excellent clinical values of this LASSO-based risk score system. Meanwhile, this risk score 
system showed better performance in the external dataset as well when comparing with nomogram. Prospective clinical trials with a larger population were needed to evaluate the performance of this model.

Several limitations should be acknowledged. First, this was a retrospective study. The prognosis of patients' survival after bronchoscopy may be associated with several factors such as the patients' general status at the intervention, the histology of the tumor, the sites and length of bronchial or tracheal stenosis. Several clinical indicators to measure symptom relief and physiological status were not included in this study. Second, the sample size in our study was still not large enough although two institutions were included. In addition, it might become more accurate to measure the degree of stenosis by interventional bronchoscopy or tools based on artificial intelligence. More parameters may be considered for analysis and these findings need to be tested in a multi-center and prospective study based on a larger population.

\section{Acknowledgments}

Funding: This study was supported in part by a grant from National Natural Science Foundation of China (81802255), Shanghai Pujiang Program (17PJD036) and a grant from Shanghai Municipal Commission of Health and Family Planning Program (20174Y0131), National key research \& development project (2016YFC0902300), Major disease clinical skills enhancement program of three year action plan for promoting clinical skills and clinical innovation in municipal hospitals, Shanghai Shen Kang Hospital Development Center Clinical Research Plan of SHDC (16CR1001A), "Dream Tutor" Outstanding Young Talents Program (fkyq1901), key disciplines of Shanghai Pulmonary Hospital (2017ZZ02012), grant of Shanghai Science and Technology Commission (16JC1405900).

\section{Footnote}

Reporting Checklist: The authors have completed the TRIPOD reporting checklist. Available at https://dx.doi. org/10.21037/tlcr-21-301

Data Sharing Statement: Available at https://dx.doi. org/10.21037/tlcr-21-301

Conflicts of Interest: All authors have completed the ICMJE uniform disclosure form (available at https://dx.doi. org/10.21037/tlcr-21-301). The authors have no conflicts of interest to declare.

Ethical Statement: The authors are accountable for all aspects of the work in ensuring that questions related to the accuracy or integrity of any part of the work are appropriately investigated and resolved. This study was conducted consistent with the provisions of Declaration of Helsinki (as revised in 2013). Approval from the ethics committee of Shanghai Pulmonary Hospital was obtained (18Q016NJ) and individual consent for this retrospective analysis was waived.

Open Access Statement: This is an Open Access article distributed in accordance with the Creative Commons Attribution-NonCommercial-NoDerivs 4.0 International License (CC BY-NC-ND 4.0), which permits the noncommercial replication and distribution of the article with the strict proviso that no changes or edits are made and the original work is properly cited (including links to both the formal publication through the relevant DOI and the license). See: https://creativecommons.org/licenses/by-nc-nd/4.0/.

\section{References}

1. Yarmus L, Mallow C, Akulian J, et al. Prospective Multicentered Safety and Feasibility Pilot for Endobronchial Intratumoral Chemotherapy. Chest 2019;156:562-70.

2. Okiror L, Jiang L, Oswald N, et al. Bronchoscopic management of patients with symptomatic airway stenosis and prognostic factors for survival. Ann Thorac Surg 2015;99:1725-30.

3. Wood DE. Management of malignant tracheobronchial obstruction. Surg Clin North Am 2002;82:621-42.

4. Stöhr S, Bolliger CT. Stents in the management of malignant airway obstruction. Monaldi Arch Chest Dis 1999;54:264-8.

5. Seijo LM, Sterman DH. Interventional pulmonology. $\mathrm{N}$ Engl J Med 2001;344:740-9.

6. Wahidi MM, Unroe MA, Adlakha N, et al. The use of electrocautery as the primary ablation modality for malignant and benign airway obstruction. J Thorac Oncol 2011;6:1516-20.

7. Dalar L, Özdemir C, Abul Y, et al. Therapeutic bronchoscopic interventions for malignant airway obstruction: A retrospective study from experience on 547 patients. Medicine (Baltimore) 2016;95:e3886. 
8. Li CH, Huang SF, Li HY. Bronchoscopic Nd-YAG laser surgery for tracheobronchial mucoepidermoid carcinoma-a report of two cases. Int J Clin Pract 2004;58:979-82.

9. Morice RC, Ece T, Ece F, et al. Endobronchial argon plasma coagulation for treatment of hemoptysis and neoplastic airway obstruction. Chest 2001;119:781-7.

10. Okada S, Yamauchi H, Ishimori S, et al. Endoscopic surgery with a flexible bronchoscope and argon plasma coagulation for tracheobronchial tumors. J Thorac Cardiovasc Surg 2001;121:180-2.

11. Moghissi K, Parsons RJ, Dixon K. Photodynamic Therapy (Pdt) for Bronchial-Carcinoma with the Use of Rigid Bronchoscope. Laser Med Sci 1992;7:381-5.

12. Ross P Jr, Grecula J, Bekaii-Saab T, et al. Incorporation of photodynamic therapy as an induction modality in nonsmall cell lung cancer. Lasers Surg Med 2006;38:881-9.

13. Bolliger CT, Sutedja TG, Strausz J, et al. Therapeutic bronchoscopy with immediate effect: laser, electrocautery, argon plasma coagulation and stents. Eur Respir J 2006;27:1258-71.

14. Cavaliere S, Foccoli P, Farina PL. Nd:YAG laser bronchoscopy. A five-year experience with 1,396 applications in 1,000 patients. Chest 1988;94:15-21.

15. Breitenbücher A, Chhajed PN, Brutsche MH, et al. Longterm follow-up and survival after Ultraflex stent insertion in the management of complex malignant airway stenoses. Respiration 2008;75:443-9.

16. Herth F, Becker HD, LoCicero J 3rd, et al. Successful bronchoscopic placement of tracheobronchial stents without fluoroscopy. Chest 2001;119:1910-2.

17. Saad CP, Murthy S, Krizmanich G, et al. Self-expandable metallic airway stents and flexible bronchoscopy: longterm outcomes analysis. Chest 2003;124:1993-9.

18. Ong P, Grosu HB, Debiane L, et al. Long-term qualityadjusted survival following therapeutic bronchoscopy for malignant central airway obstruction. Thorax 2019;74:141-56.

19. Herth FJ, Eberhardt R. Airway stent: what is new and what should be discarded. Curr Opin Pulm Med 2016;22:252-6.

20. Huang S, Xu J, An Z, et al. Clinical assessment of airway stent placement in patients with malignant airway lesions. J Thorac Dis 2018;10:3277-88.

21. Xiong XF, Xu L, Fan LL, et al. Long-term follow-up of self-expandable metallic stents in benign tracheobronchial stenosis: a retrospective study. BMC Pulm Med 2019;19:33.

22. Högerle BA, Lasitschka F, Muley T, et al. Primary adenoid cystic carcinoma of the trachea: clinical outcome of
38 patients after interdisciplinary treatment in a single institution. Radiat Oncol 2019;14:117.

23. Bhattacharyya N. Contemporary staging and prognosis for primary tracheal malignancies: a population-based analysis. Otolaryngol Head Neck Surg 2004;131:639-42.

24. Goldstraw P, Chansky K, Crowley J, et al. The IASLC Lung Cancer Staging Project: Proposals for Revision of the TNM Stage Groupings in the Forthcoming (Eighth) Edition of the TNM Classification for Lung Cancer. J Thorac Oncol 2016;11:39-51.

25. Jin F, Li Q, Li S, et al. Interventional Bronchoscopy for the Treatment of Malignant Central Airway Stenosis: An Expert Recommendation for China. Respiration 2019;97:484-94.

26. Lee SS, Shin JH, Woo CW, et al. A new model of tracheal stenosis in dogs using combined bronchoscopic electrocautery and ethanol injection. J Vasc Interv Radiol 2008;19:764-9.

27. Mancuso CA, Canfield JL, Singla D, et al. A flexible, interpretable, and accurate approach for imputing the expression of unmeasured genes. Nucleic Acids Res 2020;48:e125.

28. Tibshirani R. Regression shrinkage and selection via the lasso. J R Stat Soc Ser B Methodol 1996;58:267-88.

29. Wang Y, Li J, Xia Y, et al. Prognostic nomogram for intrahepatic cholangiocarcinoma after partial hepatectomy. J Clin Oncol 2013;31:1188-95.

30. Dasgupta A, Dolmatch BL, Abi-Saleh WJ, et al. Selfexpandable metallic airway stent insertion employing flexible bronchoscopy: preliminary results. Chest 1998;114:106-9.

31. Lemaire A, Burfeind WR, Toloza E, et al. Outcomes of tracheobronchial stents in patients with malignant airway disease. Ann Thorac Surg 2005;80:434-7; discussion 437-8.

32. Casal RF, Iribarren J, Eapen G, et al. Safety and effectiveness of microdebrider bronchoscopy for the management of central airway obstruction. Respirology 2013;18:1011-5.

33. Ost DE, Ernst A, Grosu HB, et al. Therapeutic bronchoscopy for malignant central airway obstruction: success rates and impact on dyspnea and quality of life. Chest 2015;147:1282-98.

34. Oviatt PL, Stather DR, Michaud G, et al. Exercise capacity, lung function, and quality of life after interventional bronchoscopy. J Thorac Oncol 2011;6:38-42.

35. Ortiz-Comino RM, Morales A, López-Lisbona R, et al. 
Silicone Stent Versus Fully Covered Metallic Stent in Malignant Central Airway Stenosis. Ann Thorac Surg 2021;111:283-9.

36. Ost DE, Shah AM, Lei X, et al. Respiratory infections increase the risk of granulation tissue formation following airway stenting in patients with malignant airway obstruction. Chest 2012;141:1473-81.

37. Ranu H, Evans J, Sheth A, et al. Removal of long-term tracheal stents with excellent tracheal healing. Ann Thorac Surg 2010;89:598-9.

38. Chhajed PN, Somandin S, Baty F, et al. Therapeutic bronchoscopy for malignant airway stenoses: choice of modality and survival. J Cancer Res Ther 2010;6:204-9.

39. Ernst A, Simoff M, Ost D, et al. Prospective risk-adjusted morbidity and mortality outcome analysis after therapeutic bronchoscopic procedures: results of a multi-institutional outcomes database. Chest 2008;134:514-9.

40. Amjadi K, Voduc N, Cruysberghs Y, et al. Impact of interventional bronchoscopy on quality of life in malignant airway obstruction. Respiration 2008;76:421-8.

41. Razi SS, Lebovics RS, Schwartz G, et al. Timely airway stenting improves survival in patients with malignant central airway obstruction. Ann Thorac Surg 2010;90:1088-93.

42. Maurichi A, Miceli R, Eriksson H, et al. Factors Affecting Sentinel Node Metastasis in Thin (T1) Cutaneous Melanomas: Development and External Validation of a Predictive Nomogram. J Clin Oncol 2020;38:1591-601.

43. Touijer K, Scardino PT. Nomograms for staging, prognosis, and predicting treatment outcomes. Cancer 2009;115:3107-11.

Cite this article as: Jiang $M, \mathrm{Xu} \mathrm{H}, \mathrm{Yu} D$, Yang $\mathrm{L}, \mathrm{Wu} W$, Wang H, Sun H, Zhu J, Zhao W, Fang Q, Yu J, Chen P, Wu S, Zheng Z, Zhang L, Hou L, Zhang H, Gu Y, He Y. Risk-score model to predict prognosis of malignant airway obstruction after interventional bronchoscopy. Transl Lung Cancer Res 2021;10(7):3173-3190. doi: 10.21037/tlcr-21-301 


\section{Supplementary}

Table S1 Clinical characteristics of all patients enrolled

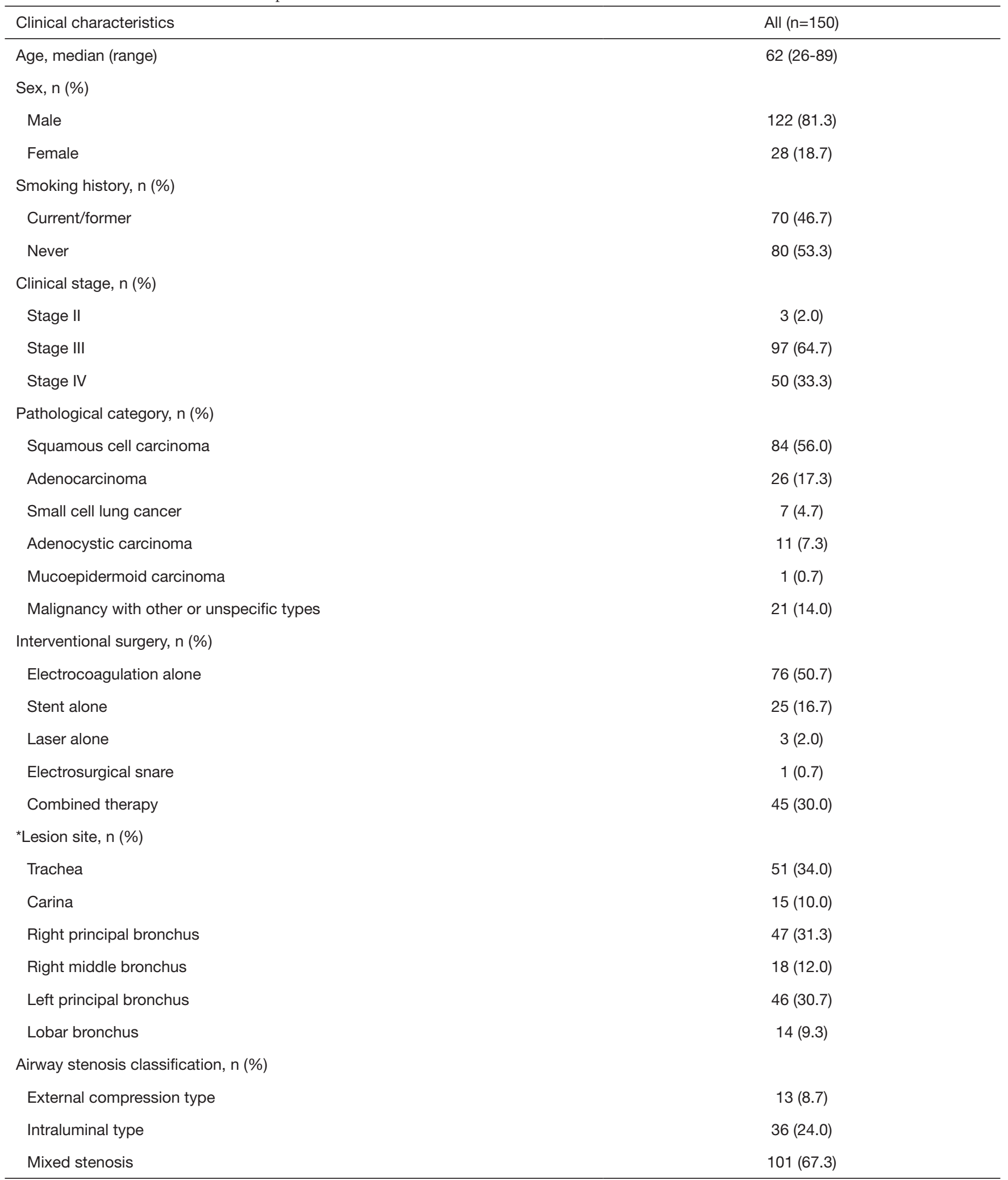

The "*” indicates patients with more than one surgical site. 
Table S2 Clinical characteristics of the cohort for studying short and intermediate efficacy

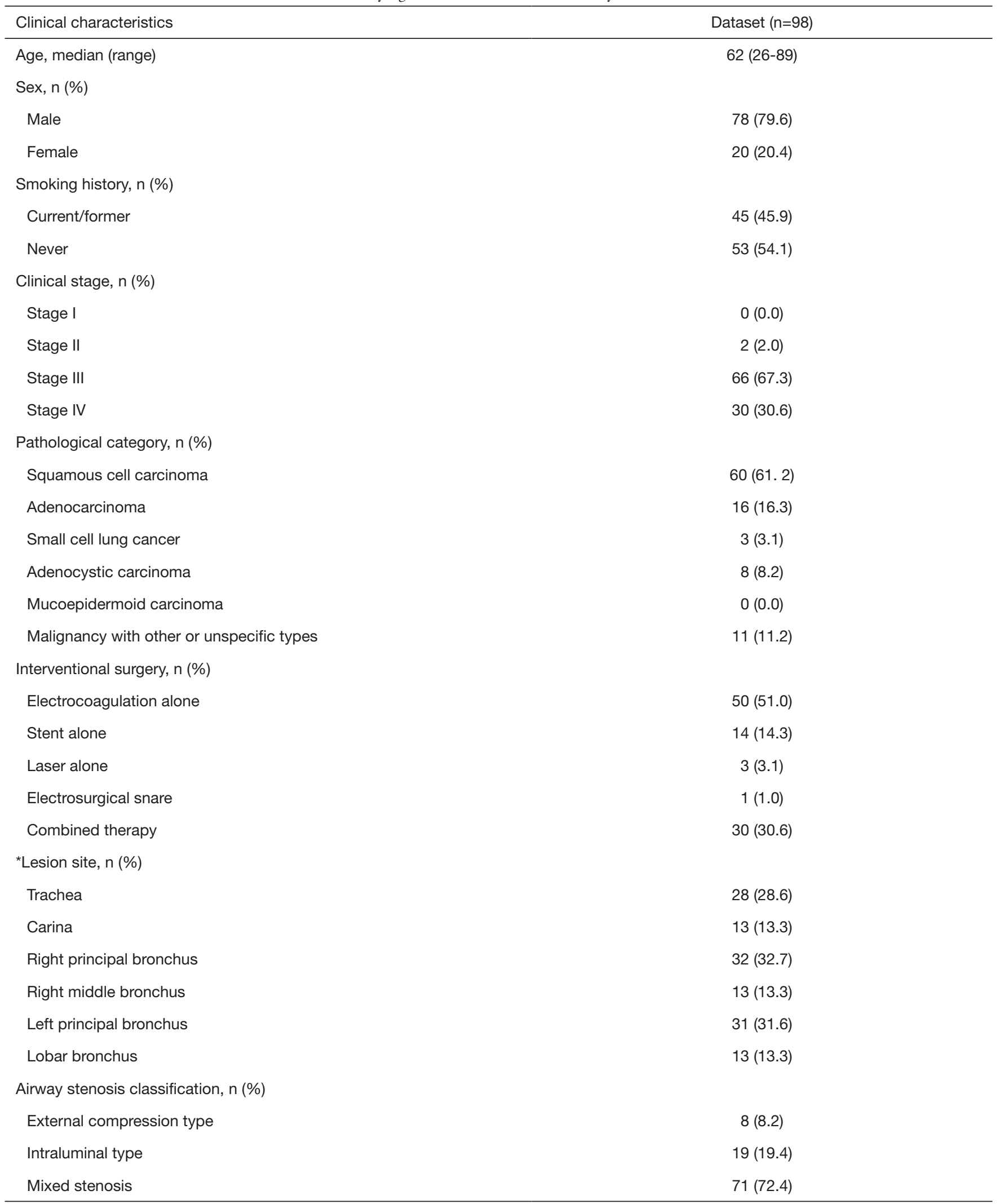

The "*” indicates patients with more than one surgical site. 
Table S3 Regression analysis for short and intermediate benefits

\begin{tabular}{|c|c|c|c|}
\hline Variables & \multicolumn{3}{|c|}{ Univariate } \\
\hline Age $(<65$ y vs. $\geq 65$ y) & 2.040 & $0.860-4.837$ & 0.106 \\
\hline Sex (female vs. male) & 0.825 & $0.293-2.328$ & 0.717 \\
\hline Smoking status (nonsmoker vs. smoker) & 1.398 & $0.595-3.284$ & 0.442 \\
\hline Stenosis classification & & & 0.395 \\
\hline Endobronchial vs. mix & 1.522 & $0.491-4.717$ & 0.467 \\
\hline Extrinsic vs. mix & 3.804 & $0.443-32.697$ & 0.223 \\
\hline Interventional bronchoscopy & & & 0.520 \\
\hline Electrocoagulation + electrosurgical snare vs. electrocoagulation & 1.111 & $0.348-3.543$ & 0.859 \\
\hline $\begin{array}{l}\text { Electrocoagulation + stenting + electrosurgical snare vs. } \\
\text { electrocoagulation }\end{array}$ & 1076983228.567 & 0.000-undefined & 0.999 \\
\hline
\end{tabular}

A.
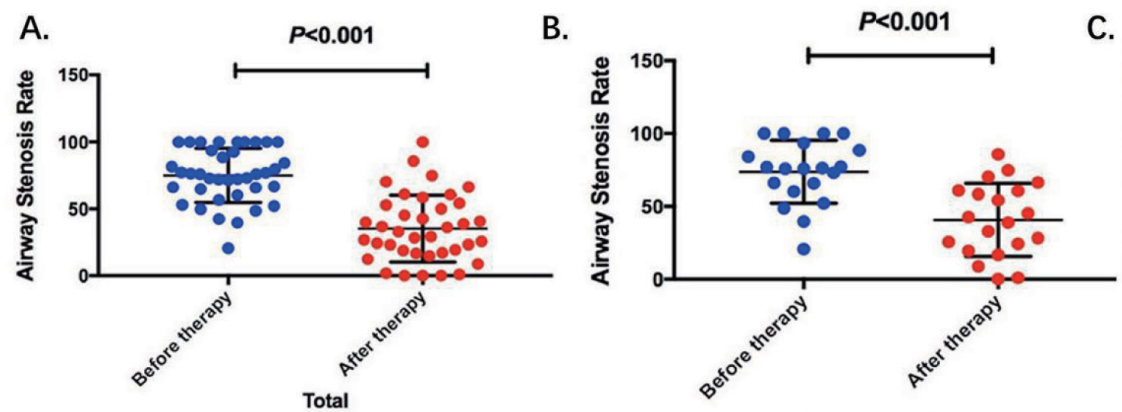

Electrocoagulation

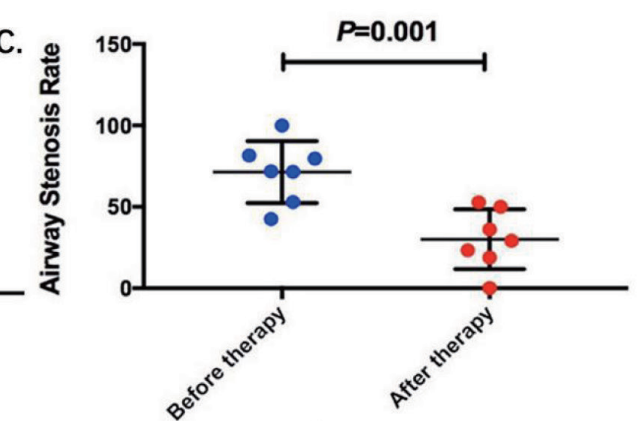

Stent

Figure S1 Short-term benefit. A significant difference of stenosis rate was observed before and after receiving treatment of (A) interventional bronchoscopy, (B) electrocoagulation, and (C) stenting in 38 MAO patients. MAO, malignant airway obstruction. 

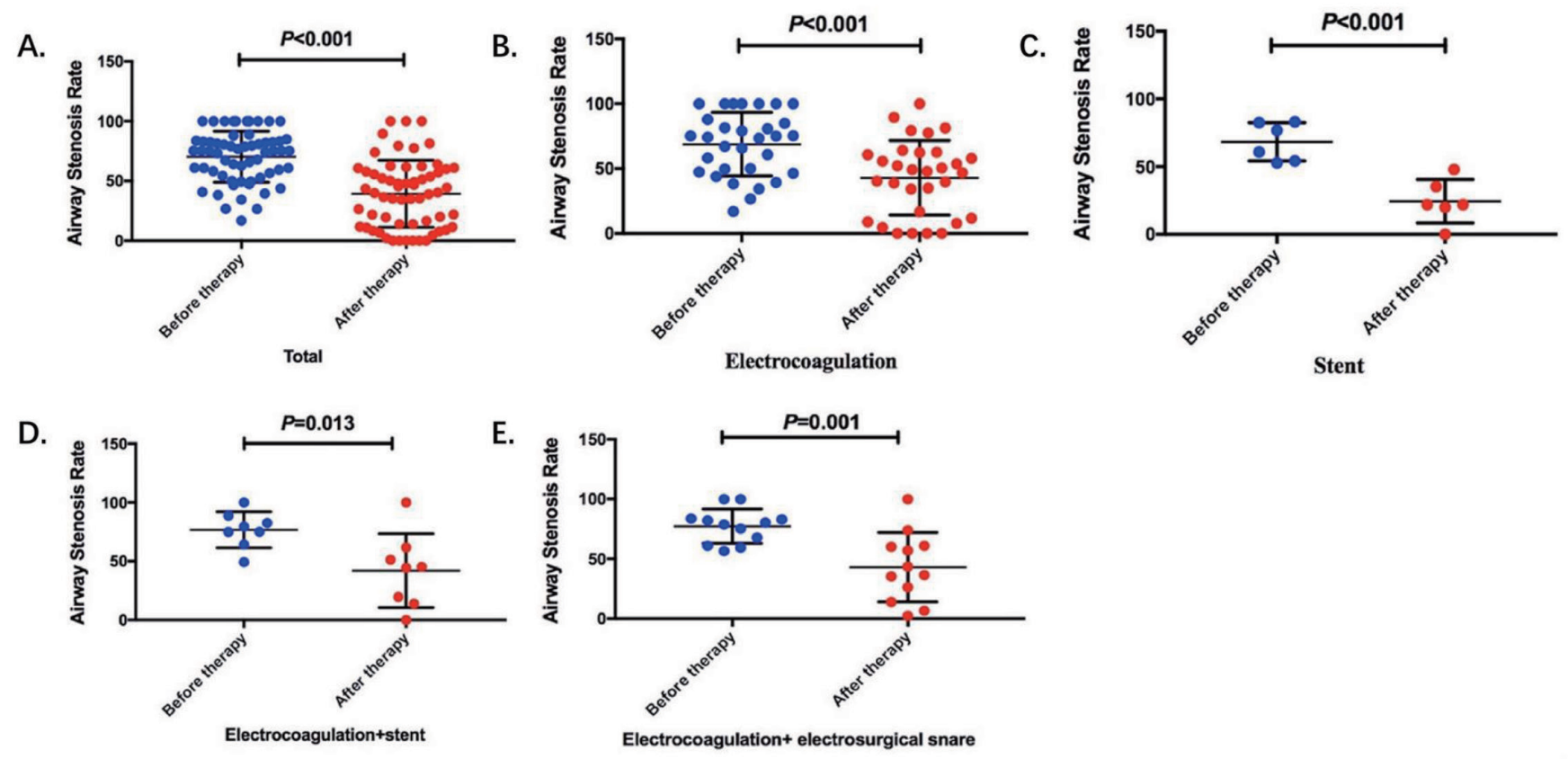

Figure S2 Intermediate benefit. A significant difference of stenosis rate was observed before and after receiving treatment of (A) interventional bronchoscopy, (B) electrocoagulation, (C) stenting, (D) electrocoagulation + stenting, and (E) electrocoagulation + electrosurgical snare in $60 \mathrm{MAO}$ patients. MAO, malignant airway obstruction.
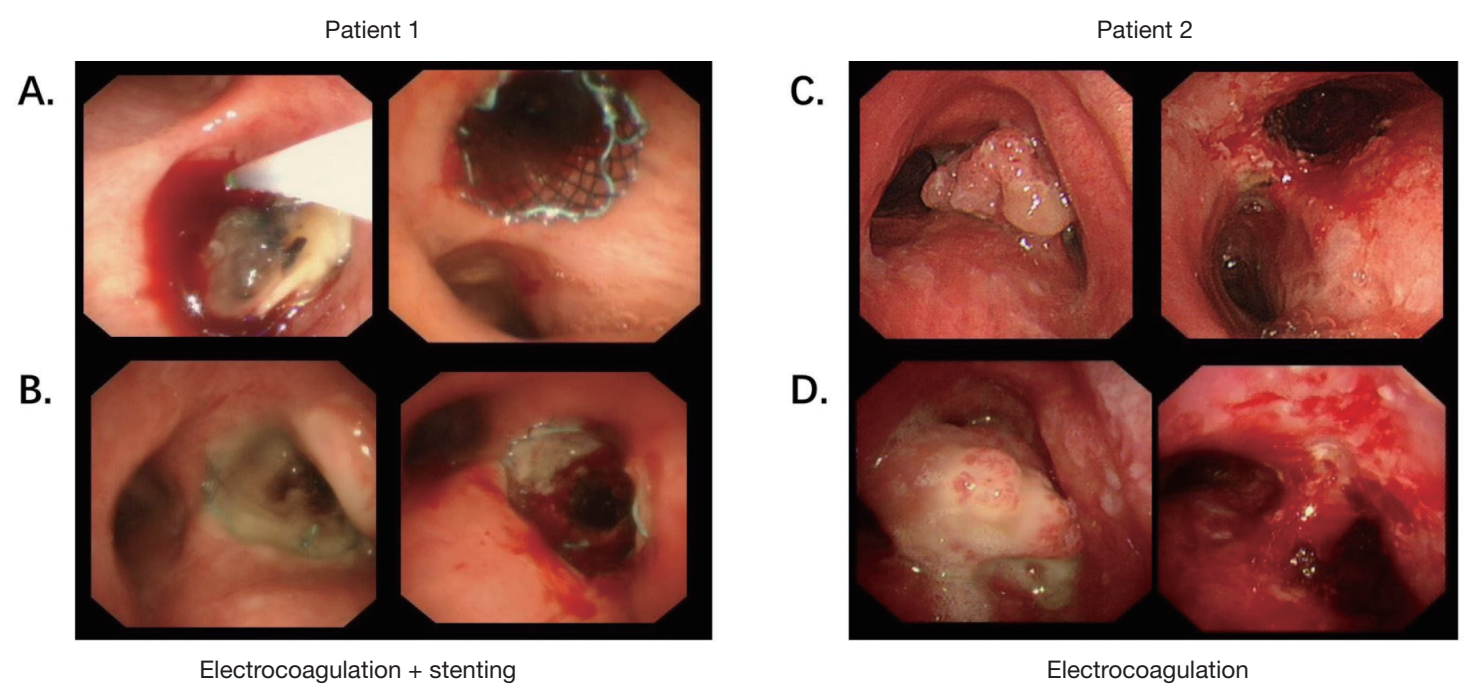

Figure S3 Lumen restenosis. For patient 1 and 2, Figure (A and C) were the diagrams before and after the first bronchoscopy treatment, which showed that the operations were very successful (A,C). However, with continuous follow-up, lumen restenosis was observed, and the second therapy was further performed (B and D). 
Table S4 Correlation of clinical factors and OS in MAO $(n=112)$

\begin{tabular}{|c|c|c|c|c|}
\hline \multirow{2}{*}{ Characteristic } & \multicolumn{2}{|c|}{ Log-rank (Mantel-Cox) } & \multicolumn{2}{|c|}{ Median survival } \\
\hline & Chi square & $P$ value & value & $95 \% \mathrm{Cl}$ \\
\hline Age & 8.297 & 0.004 & 614.000 & 269.876-958.124 \\
\hline$\geq 65$ & & & 288.000 & $121.514-454.486$ \\
\hline Gender & 1.778 & 0.182 & 614.000 & 269.876-958.124 \\
\hline Male & & & 469.000 & $281.883-656.117$ \\
\hline Smoking status & 7.945 & 0.005 & 614.000 & 269.876-958.124 \\
\hline Non-smoker & & & 905.000 & $555.485-1254.515$ \\
\hline Smoker & & & 396.000 & $212.888-579.112$ \\
\hline Stage & 0.535 & 0.465 & 614.000 & 269.876-958.124 \\
\hline Stenosis site & 3.664 & 0.056 & 614.000 & 269.876-958.124 \\
\hline Trachea and carina & & & 905.000 & 593.269-1216.731 \\
\hline Bronchus & & & 411.000 & $231.062-590.938$ \\
\hline Airway stenosis classification & 5.964 & 0.051 & 614.000 & 269.876-958.124 \\
\hline Endobronchial & & & 1065.000 & Undefined \\
\hline Extrinsic & & & 248.000 & 179.803-316.197 \\
\hline Mixed & & & 532.000 & $321.001-742.999$ \\
\hline Preoperative stenosis rate & 7.410 & 0.006 & 614.000 & 269.876-958.124 \\
\hline Low & & & 836.000 & $520.704-1151.296$ \\
\hline No & & & 614.000 & $269.443-958.557$ \\
\hline Chemotherapy after interventional bronchoscopy & 0.099 & 0.753 & 614.000 & 269.876-958.124 \\
\hline Yes & & & 836.000 & $234.825-1437.175$ \\
\hline No & & & 614.000 & $363.274-864.726$ \\
\hline Radiotherapy after interventional bronchoscopy & 2.071 & 0.150 & 614.000 & 269.876-958.124 \\
\hline Yes & & & 836.000 & $421.277-1250.723$ \\
\hline No & & & 428.000 & $154.464-701.536$ \\
\hline $\begin{array}{l}\text { Targeted therapy after interventional } \\
\text { bronchoscopy }\end{array}$ & 1.860 & 0.173 & 614.000 & 269.876-958.124 \\
\hline Yes & & & Undefined & Undefined \\
\hline No & & & 614.000 & $278.940-949.060$ \\
\hline Immunotherapy after interventional bronchoscopy & 2.110 & 0.146 & 614.000 & 269.876-958.124 \\
\hline Yes & & & 178.000 & $167.735-188.265$ \\
\hline No & & & 816.000 & $451.647-1180.353$ \\
\hline
\end{tabular}

OS, overall survival; MAO, malignant airway obstruction. 
Table S5 Clinical characteristics of the external validation cohort

\begin{tabular}{|c|c|}
\hline Clinical characteristics & All $(n=33)$ \\
\hline Age, median (range) & $65(45-83)$ \\
\hline \multicolumn{2}{|l|}{ Sex, n (\%) } \\
\hline Female & $6(18.2)$ \\
\hline \multicolumn{2}{|l|}{ Smoking history, n (\%) } \\
\hline \multicolumn{2}{|l|}{ Clinical stage, $\mathrm{n}(\%)$} \\
\hline Stage I & $0(0.0)$ \\
\hline Stage II & $1(3.0)$ \\
\hline Stage III & $17(51.5)$ \\
\hline Adenocarcinoma & $6(18.2)$ \\
\hline Small cell lung cancer & $1(3.0)$ \\
\hline Large cell lung cancer & $2(6.1)$ \\
\hline Sarcoma & $2(6.1)$ \\
\hline Mucoepidermoid carcinoma & $1(3.0)$ \\
\hline \multicolumn{2}{|l|}{ Interventional surgery, n (\%) } \\
\hline Electrocoagulation alone & $10(30.3)$ \\
\hline Stent alone & $2(6.1)$ \\
\hline Laser alone & $1(3.0)$ \\
\hline Intraluminal type & $17(51.5)$ \\
\hline Mixed stenosis & $14(42.4)$ \\
\hline \multicolumn{2}{|c|}{ Chemotherapy after interventional bronchoscopy, n (\%) } \\
\hline Yes & $11(33.3)$ \\
\hline No & $22(66.7)$ \\
\hline \multicolumn{2}{|c|}{ Radiotherapy after interventional bronchoscopy, n (\%) } \\
\hline Yes & $3(9.1)$ \\
\hline No & $30(90.9)$ \\
\hline \multicolumn{2}{|c|}{ Targeted therapy after interventional bronchoscopy, n (\%) } \\
\hline Yes & $3(9.1)$ \\
\hline No & $30(90.9)$ \\
\hline \multicolumn{2}{|c|}{ Immunotherapy after interventional bronchoscopy, n (\%) } \\
\hline Yes & $3(9.1)$ \\
\hline No & $30(90.9)$ \\
\hline
\end{tabular}

The "*” indicates patients with more than one surgical site. 
Table S6 Correlation of clinical factors and OS in the external validation cohort

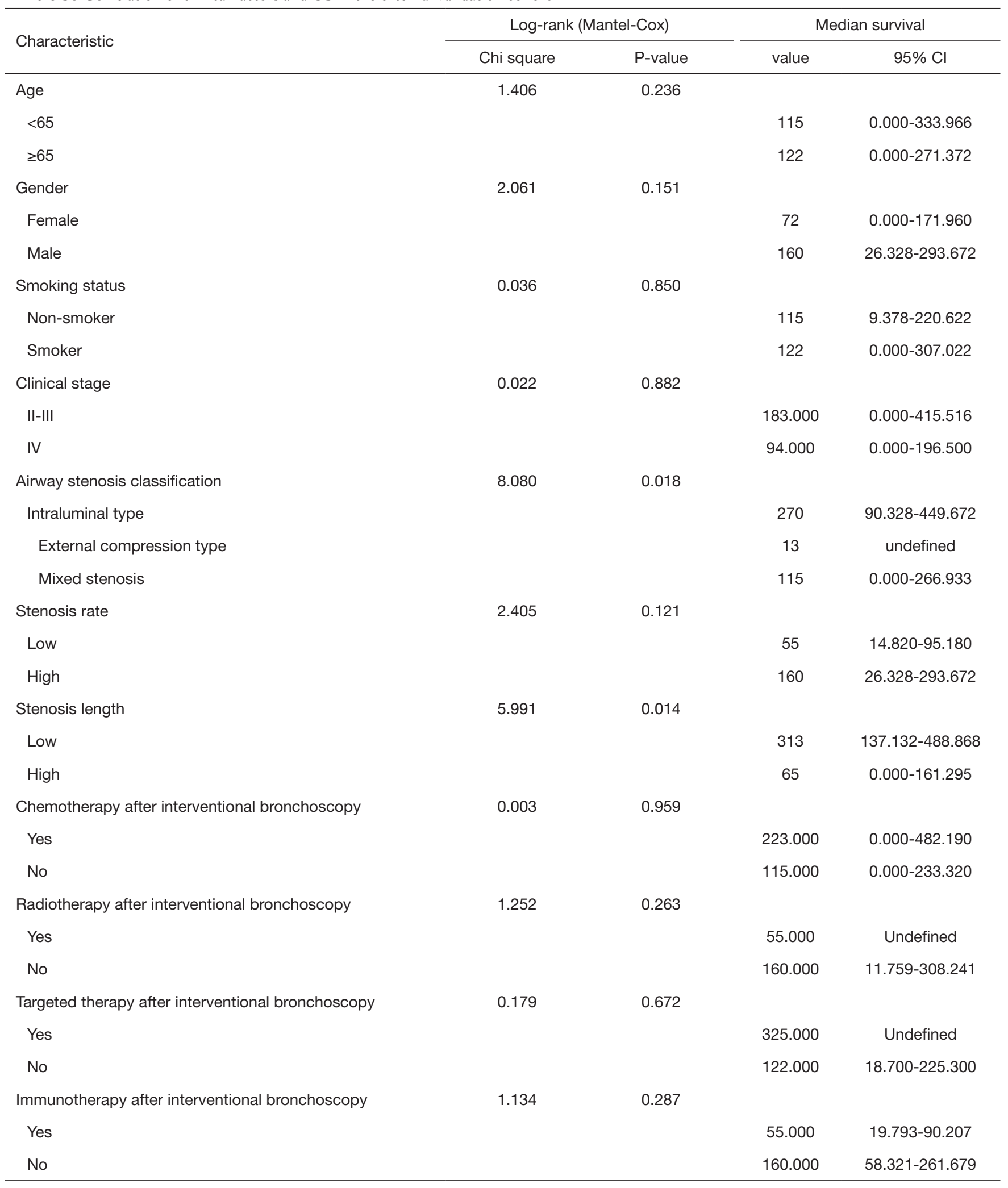

OS, overall survival. 